\title{
Developmental Design of Synthetic Bacterial Architectures by Morphogenetic Engineering
}

\author{
Jonathan Pascalie, ${ }^{*, \dagger, \ddagger, \uparrow}$ Martin Potier, ${ }^{\ddagger}$ Taras Kowaliw, $^{\dagger}$ Jean-Louis Giavitto, ${ }^{\S}$ Olivier Michel, ${ }^{\ddagger}$ \\ Antoine Spicher, ${ }^{\ddagger}$ and René Doursat ${ }^{*, \|, \dagger}$
}

\begin{abstract}
Synthetic biology is an emerging scientific field that promotes the standardized manufacturing of biological components without natural equivalents. Its goal is to create artificial living systems that can meet various needs in health care or energy domains. While most works are focused on the individual bacterium as a chemical reactor, our project, SynBioTIC, addresses a novel and more complex challenge: shape engineering, i.e. the redesign of natural morphogenesis toward a new kind of "developmental 3D printing". Potential applications include organ growth, natural computing in biocircuits, or future vegetal houses. To create in silico multicellular organisms that exhibit specific shapes, we construe their development as an iterative process combining fundamental collective phenomena such as homeostasis, patterning, segmentation, and limb growth. Our numerical experiments rely on the existing Escherichia coli simulator Gro, a physico-chemical computation platform offering reaction-diffusion and collision dynamics solvers. The synthetic "bioware" of our model executes a set of rules, or "genome", in each cell. Cells can differentiate into several predefined types associated with specific actions (divide, emit signal, detect signal, die). Transitions between types are triggered by conditions involving internal and external sensors that detect various protein levels inside and around the cell. Indirect communication between bacteria is relayed by morphogen diffusion and the mechanical constraints of 2D packing. Starting from a single bacterium, the overall architecture emerges in a purely endogenous fashion through a series of developmental stages, inlcuding proliferation, differentiation, morphogen diffusion and synchronization. The genome can be parametrized to control the growth and features of appendages individually. As exemplified by the $\mathrm{L}$ and $\mathrm{T}$ shapes that we obtain, certain precursor cells can be inhibited while others can create limbs of varying size ("divergence of the homology"). Such morphogenetic phenotypes open the way to more complex shapes made of a recursive array of core bodies and limbs and, most importantly, to an evolutionary developmental ("evo-devo") exploration of unplanned functional forms.
\end{abstract}

\section{Keywords}

morphogenetic engineering, artificial life, multicellular self-organization, agent-based modeling, physico-chemical simulation, developmental 3D printing

\footnotetext{
${ }^{\dagger}$ Complex Systems Institute, Paris Ile-de-France (ISC-PIF), CNRS UPS3611, Paris, France

${ }_{\ddagger}^{\ddagger}$ Algorithmic, Complexity and Logic Laboratory (LACL), Université Paris-Est Créteil, France

"Computer Science Research Institute (IRIT), CNRS UMR5505, Université de Toulouse, France

${ }^{\S}$ Institute for Research and Coordination Acoustic/Music (IRCAM), CNRS UMR9912, Paris, France

"Informatics Research Centre (IRC), Manchester Metropolitan University, Manchester M1 5GD, UK
} 
Background and Motivation. . . . . . . . . . . . . . . . . . 3

From the Individual Cell to Patterns. . . . . . . . . . . . . . . . . . 4

The Perspective from Artificial Development. . . . . . . . . . . . . . 5

m MORPHOGENETIC SYNTHETIC BIOLOGY . . . . . . . . . . . . 7

口 RESUlTS . . . . . . . . . . . . . . . . . . . . 12

Homeostatic Core: MorphoBrick 1. . . . . . . . . . . . . . . . . . . 12

MorphoBrick-1 Homeostatic Development. . . . . . . . . . . . . . . 12

MorphoBrick-1 Sensitivity Analysis. . . . . . . . . . . . . . . . . 15

MorphoBrick-1 Genomic Variations: Transition Thresholds. . . . . . . 17

MorphoBrick-1 Environmental Variations: Chemical Rates. . . . . . . 18

MorphoBrick-1 Genomic Mutation: Number of Types. . . . . . . . . . 18

Limb Growth: MorphoBrick 2. . . . . . . . . . . . . . . . . . . . . 20

MorphoBrick-2 Sensitivity Analysis. . . . . . . . . . . . . . . 22

MorphoBrick-2 Genomic Variations: Transition Thresholds. . . . . . . 24

MorphoBrick-2 Environmental Variations: Chemical Rates. . . . . . . 25

Precursor Cells and Assembly: MorphoBrick 3. . . . . . . . . . . . . 25

MorphoBrick-3 Segmentation. . . . . . . . . . . . . . . . . 26

MorphoBrick-3 Precursor Cell Positioning. . . . . . . . . . . . . . . 27

Class-I Shapes: Radially Symmetric Wheels. . . . . . . . . . . . . . 29

MorphoBrick-3 Precursor Cell Differentiation. . . . . . . . . . . . . 29

Class-II Shapes: Asymmetric Wheels. . . . . . . . . . . . . . . . . . 32

DISCUSSION . . . . . . . . . . . . . . . . . . 32

Robustness of Leader Singularity. . . . . . . . . . . . . . . . . . . . 34

Multiplicity of Chemical Species. . . . . . . . . . . . . . . . 35

Synchrony of Bacterial Population. . . . . . . . . . . . . . . . . 36

Midplane Feasibility. . . . . . . . . . . . . . . . . . . . 36

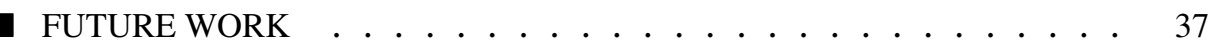

Toward Higher-Level Classes. . . . . . . . . . . . . . . . . . . 37

Polymorphism, Polyphenism and Evolution. . . . . . . . . . . . . . . 38

METHODS ........................ . . . 39

Model of Bacterial Behavior Ontology. . . . . . . . . . . . . . . . . 39

Genomic Representation. . . . . . . . . . . . . . . . . . . . . . . 40

Higher-Level Biological Functions. . . . . . . . . . . . . . . . . . . 43

Hierarchical Composition. . . . . . . . . . . . . . . . . 44

Chemical Environment. . . . . . . . . . . . . . . . . . . . . 44

YNTHETIC biology is currently in search of design principles to achieve a reliable and se$D$ cure level of functionality from reusable biological parts, as exemplified by the BioBricks framework (1). The goal is to create artificial living systems that can meet various needs in application domains such as health care, nanotechnology, energy, and chemistry. So far, most of the studies in this field have focused on the low level, seeking to characterize and validate the elementary properties of an individual bacterium. However, beyond genetic engineering problems and bioinformatics tools, computer scientists also view synthetic biology as a systems design endeavor, likened to large software systems and electronic circuits. 
In this context, our project, SynBioTIC, is positioned upstream and addresses a novel and more complex challenge at the cell population level: shape engineering, i.e. the redesign of natural morphogenesis toward a new kind of "developmental 3D printing”. Potential applications include organ growth, natural computing in biocircuits, or future vegetal houses (2) and self-repairing buildings (3). To this aim, we use realistic agent-based simulations of bacterial mats to experiment with mechanisms allowing cell assemblies to collectively self-repair and develop complex structures.

From the "bioware" viewpoint, the motivation is to exploit of the nontrivial collective properties of bacteria. From the software viewpoint, SynBioTIC proposes to design and develop formalisms and computer tools to literally "compile" (as in programming languages) the overall behavior of a population of cells into processes local to each cell. It relies on the specification of a global spatial behavior and its description across a tower of languages. Each language at a given level addresses distinct features. Its set of instructions can be compiled into the lower level, and ultimately down to the final bioware into a cellular regulation network (gene network, signaling and metabolic pathways). This "soft-to-wet" approach, similar to a classical soft-to-hard compiler, aims to fill the gap between the high-level description of a biosystem and its low-level physical requirements.

This long-term core research project is part of the broader "unconventional/natural computing" family (4), which promotes non-Turing, in materio architectures at the interface between computer science and biological engineering. It relies on the development of new approaches such as spatially explicit bacterial modeling with the Gro language (5), or more abstract "spatial computing" or "amorphous computing" with the MGS language (6) and Proto language (7), to deal with new classes of applications characterized by the emergence of a global behavior in a large population of cells that are irregularly located and dynamically interconnected.

Background and Motivation. Cameron et al. (8) propose a brief history of synthetic biology across three major periods, covering important milestones from the 1960's to this day. The authors trace the origins of the field to a publication by Jacob and Monod (9) that postulated the existence of 
genetic circuits involved in the cell's response to its environment, and already envisioned the design of new regulatory elements. However, a deeper understanding of the gene regulation machinery was still lacking in order to view synthetic biology as a true engineering challenge. With the advent of the genomic era and the rise of systems biology in the 1990's, genome sequencing and analysis became commonplace and opened the way to solving the difficult challenge of reverseengineering gene regulatory networks (GRNs) (10). By that time, it was a widely accepted notion that molecular constituents could be considered "units of computation" (11).

From the Individual Cell to Patterns. Initially, synthetic biology focused on the individual behavior of cells. The main goal was to design GRNs that could behave functionally in ways similar to electronic circuits (12). For example, Gardner et al. (13) proposed a model of "toggle switch" driving the production of two mutually inhibitory repressors, i.e. in which a cell could express either one of two proteins in response to external stimuli. Another example, the "repressilator" by Elowitz et al. (14), consists of a triple negative-feedback loop that leads the GRN to periodically induce the synthesis of green fluorescent protein as a readout of its state in individual cells. Overall, the beginnings of modern synthetic biology were guided by analogies between the fabrication of organisms and computer engineering. Notable achievements within this paradigm comprised the implementation of logic gates $(15,16)$ and a form of memory (17). These approaches, however, essentially studied the behavior of local genetic circuits in single cells without aiming toward the design of collective multigene and/or multicellular function. As pointed out by Palsson (18), in silico biology needs to move from a reductionist paradigm to one that views cells as systems and agents in interaction.

An important step toward the development of actual multicellular behavior was the engineering of cell-cell communication modules in E. coli (19). With this, a first achievement of collective function design was the creation of a homeostatic bacterial population by coupling gene expression and cell death through "quorum sensing" (20). Attention also turned to the spatial extension of cell populations. In a seminal work on static pattern formation by Basu et al. (21), sender and 
receiver bacteria evolve together on the proliferation medium. Cellular differentiation is based on a chemical gradient synthesized by sender cells, while receiver cells respond to a range of chemical concentrations and form ring-like patterns. Tabor et al. (22) proposed an edge-detection algorithm genetically encoded into an isogenic community of E. coli sensing an image of light. Communication among bacteria allows them to identify the light-dark transition edges and present the result of the computation visually. More recently, Liu et al. (23) built a synthetic genetic circuit that couples density and motility and enables the sequential self-formation of periodic stripes of high and low E. coli cell densities.

As argued by Amos (24), synthetic biology will be undergoing a "third wave", marking its progression from a single-cell approach to a population approach - a trend he likens to the past expansion from individual computers to the Internet. Following this direction, the present work aims to push the exploration of spatial self-assembly further by bridging the gap between synthetic biology and artificial morphogenesis. Generally, a clear distinction can be drawn between two major types of form-creating complex systems: ones that display simple repetitive patterns (spots and stripes), and ones that produce sophisticated functional forms (bodies and constructions). At the time of his famous paper on the chemical basis of morphogenesis, Turing (25) was already well aware of this qualitative difference, as he is said to have quipped: "The stripes are easy, it's the horse part that troubles me". To pursue Turing's "zebra" challenge in synthetic biology, we present here a methodology based on realistic in silico simulations of structured self-organization in bacterial mats. This proof of concept should constitute the basis for a future real-world implementation in bioware.

The Perspective from Artificial Development. Doursat et al. (26, 27) propose a four-part classification of the field of Morphogenetic Engineering, based on the type of self-assembly process that produces a top-level architecture or organism: "constructing" systems, in which a few agents build a precise, relatively sparse structure (as in modular robotics); "coalescing" systems, in which large flocks or swarms of agents create certain patterns or adopt global shapes (mostly in simula- 
tion); "developing" systems, in which agents are recursively added by division or aggregation to an initial seed or group; and "generating" systems, in which parts are rewritten, i.e. replaced by others based on a grammar (as in L-systems). The work presented here belongs to the third category, which refers to a recent avenue of bio-inspired works such as "artificial ontogeny" (28), "artificial embryogeny" (29, 30), "embryomorphic engineering" $(31,32)$ or "in silico evo-devo" (33), all taking multicellular development as a model and aiming to grow artificial structures starting from a single cell or a few cells.

From the viewpoint of evolutionary computation (genetic algorithms, genetic programming), developing systems do not rely on a "direct encoding" of their morphology in the genome, but an indirect encoding in the parameters of the growth process. In this first version of our work, the genotype-to-phenotype mapping is fixed and parameters contain the necessary information to achieve a specific shape. In a later stage, we intend to reintroduce evolution and combine it with rational design to create new shapes. Beyond computer simulations, ideas about developing systems were also partially realized in hardware, or “roboware”, such as Rubenstein's Kilobot swarm (34). Ultimately, our goal here is also to return to in materio computation in the biological substrate and, in a feedback loop, re-engineer morphogenesis into multicellular assemblies—whether prokaryotic species not usually forming complex structures, or eukaryotic species that could be programmed differently, such as plant cells to grow buildings or animal cells to grow organs. In sum, morphogenetic synthetic biology could be described as as a form of "bio-inspired bioware".

To set up the reverse-engineering chain going from a high-level specification of a structure all the way down to the generation of local cellular components, we need to solve fundamental questions pertaining to the representation of a phenotype by a genotype, which are also shared by swarm robotics and spatial/amorphous computing systems. Defining the morphology of an artificial creature or robot through its building blocks has been a classical approach for the past two decades. In that category, Komosinski's Framsticks (35) were an early attempt to specify complex organisms from simple sticks. In these examples, however, the specification of the structure relied on direct encoding in a text file-which made it easy for a human operator to modify, or possibly 
for evolution to create high-level mutations, but not for any developmental process to take place. On that basis, Lipson and Pollack's Golem project (36) showed that it was possible to cross the reality gap: actual robots were created following the techniques tested and optimized in simulation. First, morphologies and controllers were evolved in a virtual environment, then the best individuals were realized mechanically and immersed in the real world. Together, these works proved the suitability of in silico experiments as an "engineering wizard", even though their building blocks were not self-made via a growth process.

Artificial development is now of great interest to robot design and mostly studied in simulation $(27,37,38)$, since physical 3D self-assembling systems are still rare. In these works, multicellular development is used as a means to produce fine-grained morphologies and improve them by evolution. From the first steps made by Eggenberger (39) to the latest production of Disset et al. (40), which uses continuous cell shapes in a continuous medium, morphological complexity has greatly increased. Nevertheless, three main components remain: stigmergic interactions via chemical diffusion and gradient-based chemotaxis; gene regulation triggering differentiation into types; and cell division, motion and death.

\section{口 MORPHOGENETIC SYNTHETIC BIOLOGY}

Current applications of synthetic biology, which focus on the individual bacterium as a chemical reactor, come from biochemistry and cellular biology. The main types of applications are the production of useful molecules and materials (drugs, biofuels, bioplastics) and the detection of specific conditions in the environment, for example by changing color (biosensors). The shape engineering challenge of SynBioTIC, for its part, belongs to "morphogenetic engineering" (ME), a field founded by Doursat et al. $(26,27)$ which studies the transfer of natural morphogenesis to the design of the self-organizing abilities of the elements of complex systems. Generally, natural pattern formation (stripes, spots, waves, branches) is stochastic and repetitive, in the sense that the characteristic scale of the motifs can be known but not exact their position, whereas elaborate devices are the deterministic product of human design. Yet, multicellular biological organisms are striking ex- 
amples of complex systems that are both entirely self-organized and strongly architectural, as they display a precise arrangement of parts (41). Accordingly, ME establishes a new object of research at the intersection between traditionally disconnected domains: it stresses the programmability of self-organization, underappreciated in complex systems science, and, conversely, the benefits of self-organization, which are underappreciated in engineering.

To support a wide variety of shapes in bioware, the growth process needs to be sufficiently generic and parametric at the same time. Our purpose is not to produce a few particular outcomes but provide a developmental framework capable of generating a whole family of them. In this study, we choose a high-level morphological specification based on two types of elementary multicellular structures: core bodies, also called "wheels", and limbs, also called "sticks" (Fig. 1). Network architectures made of nodes and links are a universal form of organization observable at all levels of life: gene regulation networks, brain, skeleton, branching systems (vascular, respiratory and peripheral nervous systems; virtually all plants). It embodies a building-block game that can produce rich and complex morphologies from simple primitives, especially by isotropic (wheel) and unidirectional (stick) development. Sticks can grow out of wheels at various angles and with various lengths, while wheels of various diameters can burgeon at the extremities of newly grown sticks. The set of shapes obtained by combining these building blocks can generally be described by planar graphs or "circuits", in which neighboring nodes are connected or not by an edge. Here our purpose is to control the exact geometric features of the overall phenotype, by programming them indirectly in the genotype, not just let any mesh grow randomly. We are interested in architectures, not textures.

To this aim, we propose a five-class taxonomy of wheel-and-stick shapes sorted in order of increasing morphological and developmental complexity, i.e. difficulty of design (Fig. 1A):

- Class I: Radially symmetrical degree-1 shapes. In this first class, shapes are based on a single isotropic wheel (the core body of the organism) fitted with identical sticks positioned at regular angles (its limbs), in the style of a "cross sign" or "starfish". Development proceeds in two stages: isotropic proliferation of the initial cell into the wheel of the organism, then 


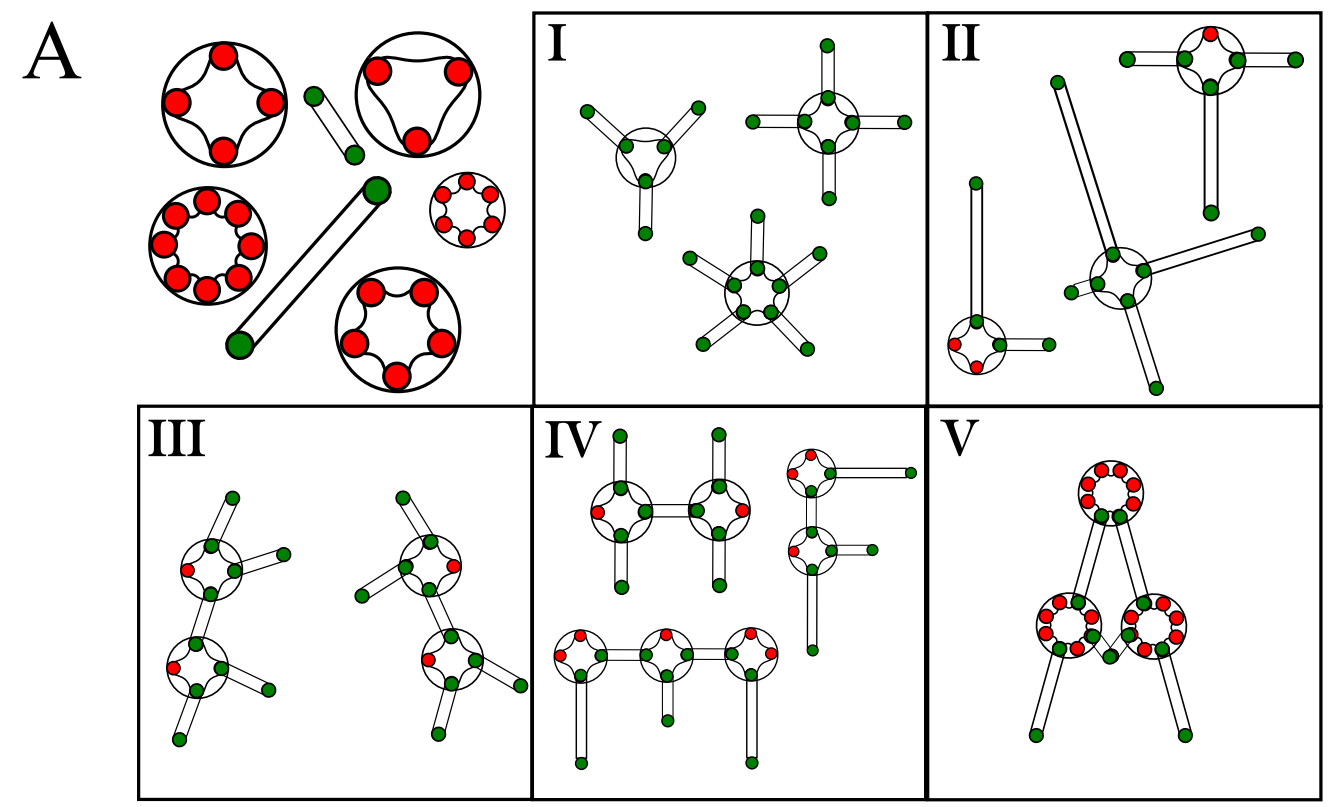

$\mathrm{B}$

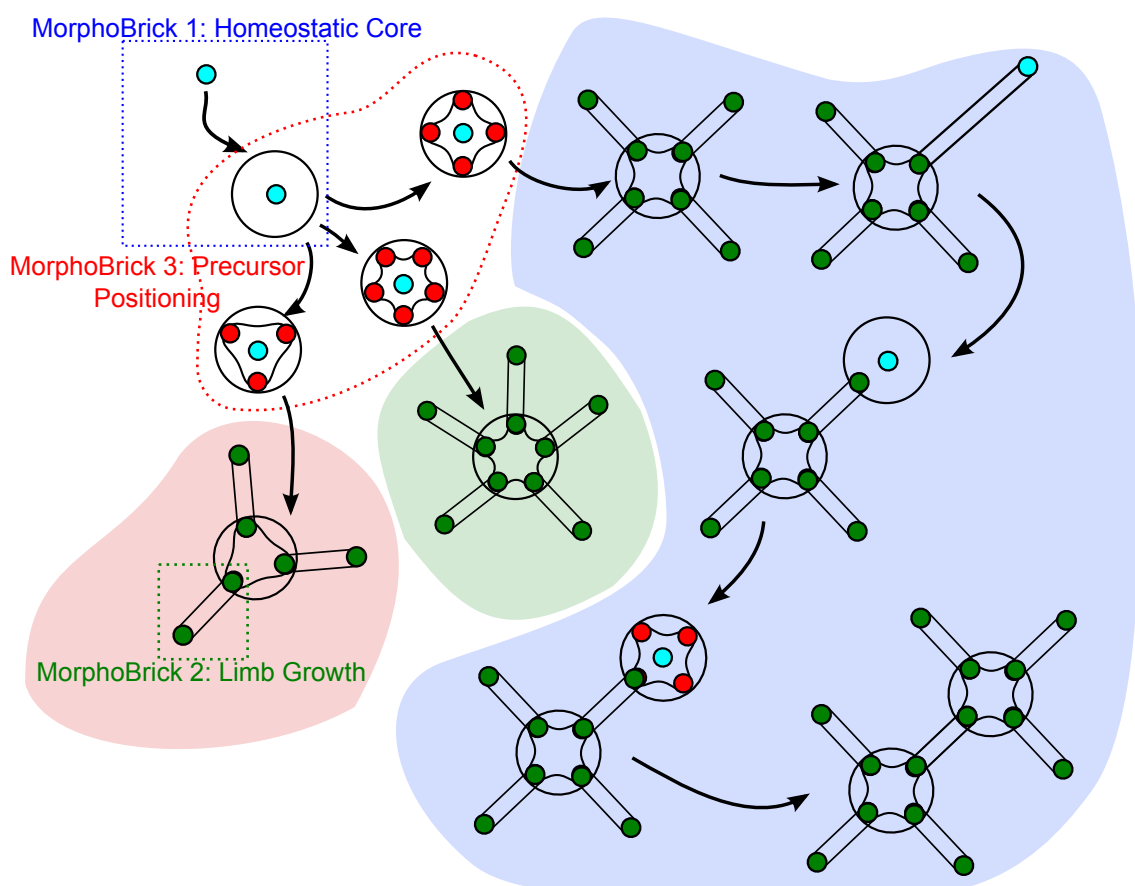

Figure 1. Targeted wheel-and-stick shapes. (A) Five-class taxonomy in increasing developmental complexity. Class I, radially symmetrical degree-1 shapes: a single wheel is fitted with identical sticks positioned at regular angles. Class II, asymmetrical degree-1 shapes: heterogeneous sticks are attached to a single polarized wheel. Class III, unaligned degree-n shapes: two or more wheels are linked by sticks, while other sticks grow at unrelated angles. Class IV, aligned degree-n shapes: sticks grow at correlated angles. Class V, cyclic degree-n shapes: sticks from different wheels can merge. (B) The three elementary "MorphoBricks" whose composition supports this variety of shapes: homeostatic core (wheel), limb growth (stick) and precursor cell (wheel-stick joint). The examples given here belong to Class I (pink and green domains) and Class IV (blue domain). Their implementation in the multicellular medium is explained in the "Results" section. 
growth of a few sticks (typically 2 to 8 ) from precursor cells that have differentiated around the crown. These precursor cells are of the same type, giving rise to individuals that exhibit "serial homology". The number, length and thickness of the sticks can be tuned in the genome.

- Class II: Asymmetrical degree-1 shapes. Here precursor cells adopt different types, hence sticks attached to a single wheel may have diverse characteristics. In some of them, stick growth can be inhibited altogether, creating a gap compared to a regular cross or star shape. Examples include letter shapes such as "T", "L", "V" and"Y". The main feature of this class is that the wheel is polarized, allowing cells to acquire positional information with respect to two main axes, anterioposterior (AP) and dorsoventral (DV) supported by four poles (north, south, east, west). More axes based on intermediate poles (northwest, etc.) can also be introduced.

- Class III: Unaligned degree-n shapes. This class represents the first level of a recursive developmental process. It comprises shapes with two or more wheels linked by sticks. Stick extremities lead to the development of wheels that initiate new sets of sticks. Although wheels can be locally polarized, they do not maintain relative orientation with respect to each other. Attached wheels only share one axis along their common stick. Typical shapes can look like articulated "snakes" (simple chains of stick segments) or "centipedes", where segments alternate with pairs of legs, possibly of different lengths.

- Class IV: Aligned degree-n shapes. Next, an additional requirement is that sticks growing on connected wheels do so at specific relative angles, for example parallel or perpendicular to each other. This is where we can design more controlled geometries such as letters with two joints ("Z", "F”) and three joints ("W”, "E”).

- Class V: Cyclic degree-n shapes. Finally, the most complex shapes of the wheel-and-stick family involve sticks of different origins crossing paths and merging into the same wheel, thereby creating cycles in the graph structure. They include characters " $\mathrm{A}$ " and " 4 ". Once 
the convergent growth of two sticks is under control, any mesh figure or circuit architecture can potentially be reached by carefully designing the genetic program. Before that level, however, the previous four classes must also be mastered and issues of computing speed resolved.

The present study does not cover this full taxonomy but explores its different levels in increasing order of complexity by model and simulation. It presents original results about the theoretical and practical possibility of (re)programming bacteria to create certain multibacterial shapes in a controlled way. Starting with Classes I and II, we progressively build the elementary components needed for higher classes: single wheel, single stick, undifferentiated then differentiated precursor cells, wheel with sticks, stick with a wheel, and so on. However, since each one of these components may include hundreds of cells, we also quickly face computational limitations that prevent us from calculating truly complex organisms. This is why our numerical experiments stop for now at the entrance of Class III, and we only describe principles and mechanisms for the rest.

The remainder of the article is organized as follows. In the "Results" section, we describe how the mapping from an abstract geometry to the multicellular medium can be realized in principle, via in silico numerical experiments. We show the development of three "MorphoBricks" (wheel, stick, and wheel-stick joint) and their composition into more complex Class-I and Class-II organismsall starting from the controlled proliferation of a single cell. Then, in the "Discussion" section, we review the simplifications made in the model and their shortcomings, particularly from the perspective of crossing the "reality gap" toward in vivo implementations". In "Future Work", we mention possible alternatives and new avenues of research worth exploring. Finally, in "Methods", we present the model of "bacterial behavior ontology" at the basis of the simulations, and the programming language that we designed to encode its associated genomic representations.

\footnotetext{
${ }^{1}$ We use "in vivo" in the synthetic-biological sense of genetic material (DNA, plasmids) implemented inside live cells, as opposed to being isolated in a test tube. Although the bacterial colony may be contained in a petri dish, it is not considered "in vitro" because it constitutes a living organism in itself.
} 


\section{- RESULTS}

Homeostatic Core: MorphoBrick 1. As presented above, the two morphological components of our shape specifications are wheels and sticks, which can be combined in a variety of ways to generate increasingly complex forms. In the next few paragraphs, we describe how these two components are able to arise from the controlled proliferation of a single cell, i.e. how the mapping from abstract geometries to the multicellular medium can be realized. In a third stage, we provide the composition mechanism between the wheel and the stick in the form of "precursor cells", a targeted differentiation mechanism on the outer rim of the wheel body by which sticks are affixed to wheels. Precursor cell positioning will be the cornerstone of the developmental process as it is responsible for building the joints between the core body and the limbs at locations specified by the morphogenetic program.

Altogether, we call MorphoBricks this set of three self-made, self-assembling components, in a reference to the concept of BioBricks (I) translated from the molecular, genotypic level to the morphological, phenotypic level (Fig. 1B). In each case, we will pay special attention to the reproducibility and programmability of the virtual growth experiments through sensitivity analysis and parametric exploration. This will also offer a glimpse of the great diversity of shapes that could theoretically be engineered through the proposed method. To this aim, we used OpenMOLE (42), a middleware platform facilitating massive experimental parametric search of complex systems models on a computing cluster, leveraging the power of the European Grid Infrastructure (EGI).

MorphoBrick-1 Homeostatic Development. Homeostasis, the process by which the internal state of a system remains constant through self-regulation, is a major characteristic of life. Therefore, homeostatic properties should be a prime concern of synthetic biology efforts aiming to build complex structures. Here our first goal is to create a "core" morphological component that maintains itself in place. This requires finding a means to maintain a stable population size. In the shape engineering challenge, the ability to control the size of a colony of bacteria over time is crucial to ensure the sustainability of their collective behavior. 
The mechanism that we propose for the development and maintenance of a homeostatic core starting from a single cell relies on a morphogenetic field emitted by a "leader cell", which could be the initial cell or not (Fig. $2 \mathrm{~B}, t_{2}$ ). There are two design strategies to ensure the unicity of the leader: either by inhibiting its mitosis, or by making it asymmetric (43), i.e. one of its two daughter cells immediately differentiates into another type, keeping the leader role in the other daughter. The problem with the latter scenario is that it creates a continual random-walk displacement of the leader at each generation, which produces a lopsided wheel shape due to the morphogen trail left by the leader's displacement (a similar effect will be in fact exploited, not suppressed, in MorphoBrick 2). Thus we opt for a nondividing leader cell, instead. A consequence is that it cannot be the initial cell and must appear later (but soon enough) via differentiation of another cell—while proliferation is ensured by the presence of a sufficient number of non-leader cells.

The genome designed for this purpose is represented graphically in Fig. 2A (see explanation in "Methods", in particular "Genomic Representation"; the full program can be found in Supporting Information, Code 1a). Initialization and leader cell generation are key mechanisms for the success of the development of this MorphoBrick and the others. The single cell of origin is in a neutral INIT state, which can differentiate into any of three active types composing the assembly: LEADER, POP1 and POP2. The emergence of the first one is a rare stochastic event; the other two depend on local morphogen concentrations, themselves resulting from emission by the cells and passive diffusion. The LEADER cell continuously secretes a chemical $\alpha$ by accumulating a fixed concentration amount $C_{\alpha}=50$, which we refer to as the "rate" of emission, in the source location at each time step (the actual concentration $[\alpha]$ reaches a plateau due to degradation and diffusion perpetually at work in every point). This triggers the differentiation of nearby cells into POP1 and creates a first circular layer of this type until a certain concentration $\theta_{\alpha}=0.8$, called differentiation threshold (controlling three transitions). Meanwhile, POP1 cells emit chemical $\beta$, which creates a second circular layer of POP2 until $\theta_{\beta}=2.5$, called survival threshold (controlling two transitions). The net result is the establishment of two stationary (but not static) rings of cell populations around the LEADER cell, each one responsible for the next. They are characterized by "fountain"-like collective motion, as 


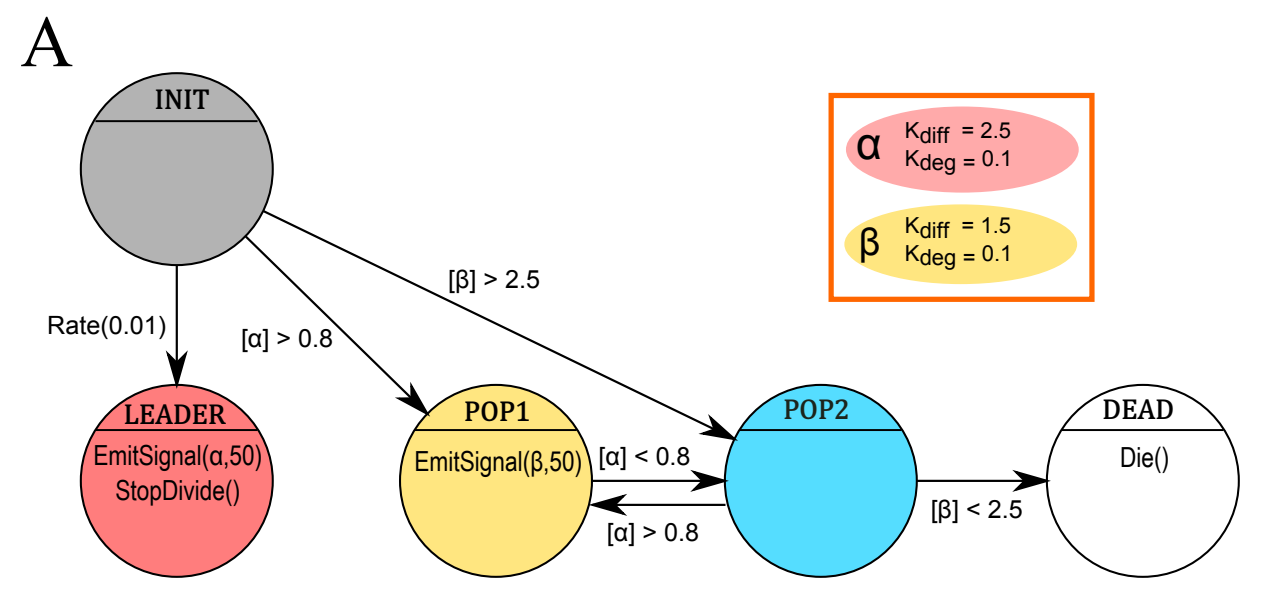

$\mathrm{B}$

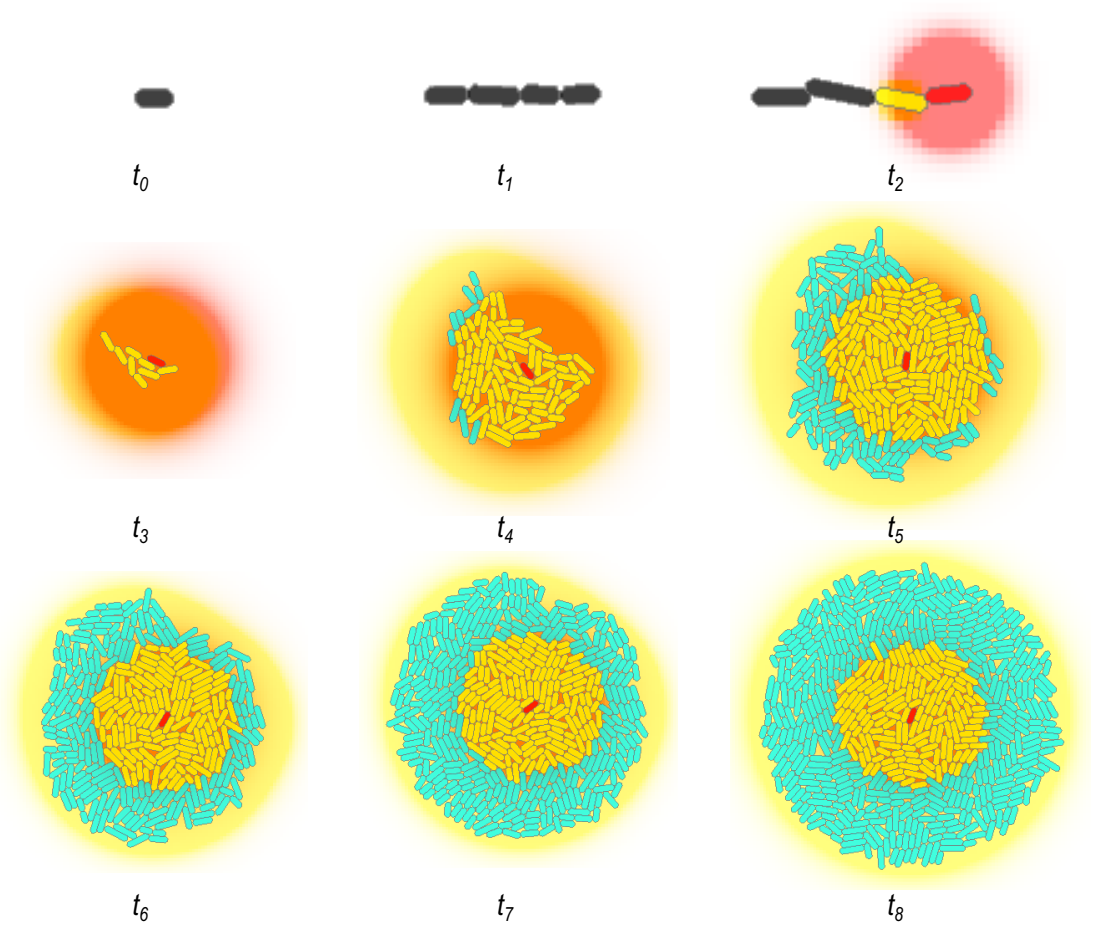

Figure 2. Genomic representation and simulation of MorphoBrick 1, "homeostatic core". (A) The differentiation graph (as in Fig. 12A) comprises four active cell types plus a DEAD type that triggers cell death. (B) Nine snapshots of the cell assembly showing the main developmental stages (Supporting Information, Video 1a). All cells except LEADER and DEAD continually divide by default. Two chemical fields, $\alpha$ and $\beta$, emitted and detected by the bacteria control their proliferation, leading to homeostasis of the assembly and maintenance of a wheel shape once a certain size is reached. The developmental scenario can be summarized as follows: The INIT type (in gray) differentiates into a LEADER cell (in red) upon a rare stochastic event (here at $t_{2}$ ). The LEADER cell secretes a diffusive morphogen field $\alpha$ (pink cloud) at rate $C_{\alpha}=50$, which causes nearby INIT cells to differentiate into POP1 (in yellow), preventing them from creating another leader. In turn, POP1 cells diffuse $\beta$ (yellow cloud), which causes the remaining INIT cells (where $[\alpha]<\theta_{\alpha}=0.8$ ) to turn into POP2 (in cyan). Moreover, POP2 cells die at the border of the $\beta$ field, where its concentration drops below $\theta_{\beta}=2.5$. In the end, a stationary (but not static) bilayered core emerges: the leader cell maintains the first layer, which maintains the second layer. 
bacteria are being pushed out by their own proliferation, becoming alternatively (and reversibly) POP1 or POP2, then eventually dying, depending on the underlying morphogenetic fields that they collectively generate.

The final size of the organism and the thickness of the layers depend on several parameters. In a given chemical environment, morphological characteristics vary with the differentiation and survival thresholds, which are features of the genome: clearly, the smaller $\theta_{\alpha}$ or $\theta_{\beta}$, the thicker the POP1 or POP2 layer, respectively. Conversely, given a certain genome, the trend would be similar if bacteria were immersed in a different chemical environment where $\alpha$ or $\beta$ had a higher diffusion rate $\kappa_{\text {diff }}$ or a smaller degradation rate $\kappa_{\text {deg. }}$. In the next paragraphs, we study quantitatively these phenotypic variations as a function of the genetic parameters and environmental conditions, starting with robustness with respect to random initialization.

MorphoBrick-1 Sensitivity Analysis. First, the invariance of the homeostatic core behavior with respect to experimental conditions is studied by repeating many times the same simulation under the same parameters, only using different random generator seeds. The main consequence is felt at the level of the INIT $\rightarrow$ LEADER transition, as the stochastic differentiation event may affect a different cell than the one in Fig. $2 \mathrm{~B}$ at $t_{2}$ and/or occur at an earlier or later time step. Other, subtler effects are caused by the fact that daughter cells are not exact clones of the mother. The Gro simulator ascribes slightly different properties to bacteria when they divide, such as their growth rate, by drawing them from Gaussian distributions (it also models fluctuations through a variable $d t$ obeying a Gamma distribution), which produces bacteria colonies of variable size and layout.

In any case, we analyze the outcome of the developmental process based on three main geometric measures: the total radius of the whole organism, the inner radius of the inner layer, and their difference, the crown thickness (outer layer's radius). The global radius is simply the average of the four distances that separate the LEADER cell from the left-most, right-most, top-most and bottom-most bacteria (all cell center coordinates being known). The inner radius is obtained in the same way, counting only the POP1 cells. Fig. 3A shows the distributions of these three quantities 

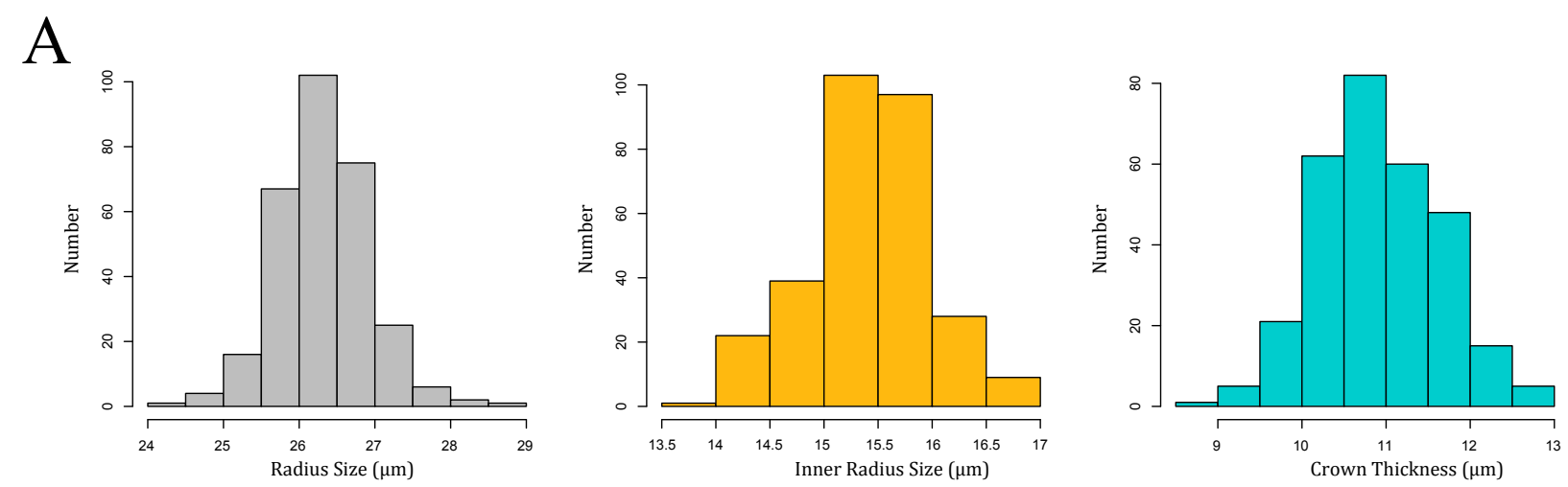

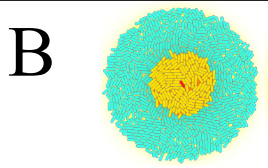

(a)

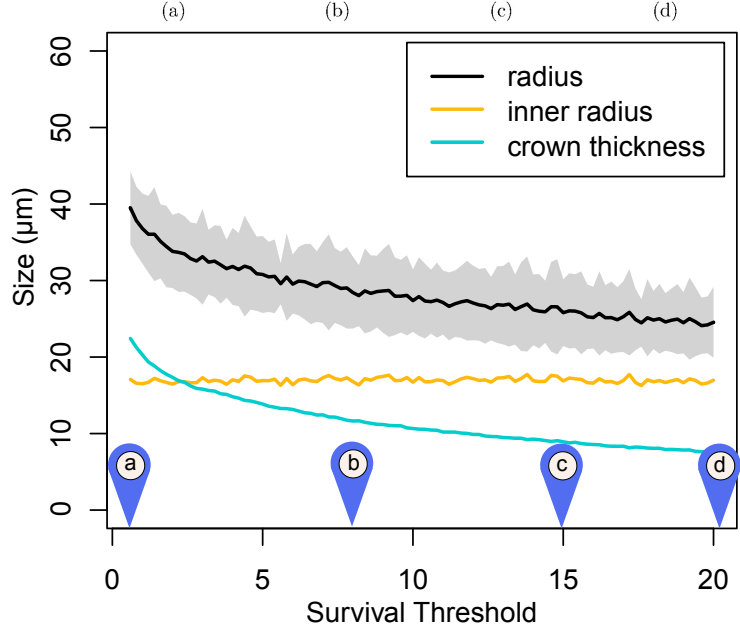

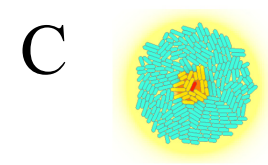

(a)

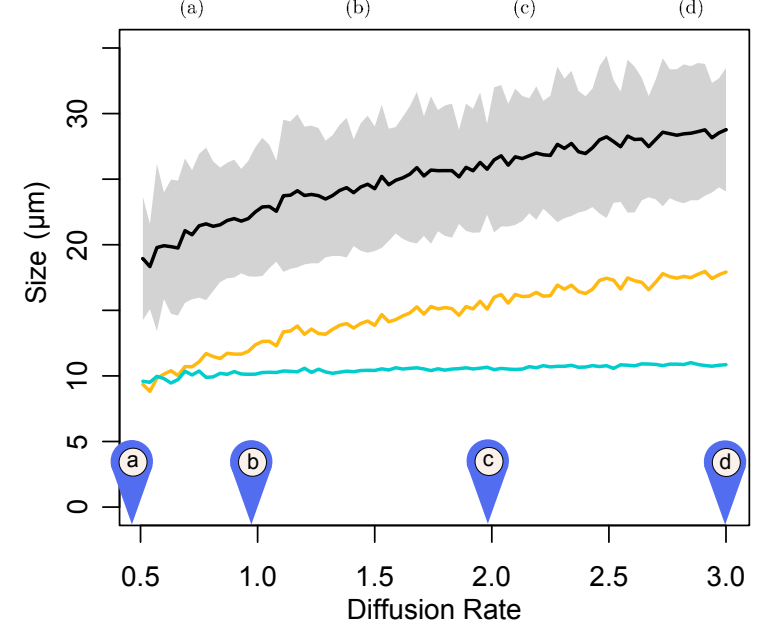

Figure 3. Sensitivity analysis and parametric exploration of MorphoBrick 1, "homeostatic core". (A) Distributions of three morphological characteristics, left to right: outer radius, inner radius, and their difference, the crown thickness. All radial sizes were measured directly in the Gro environment based on minimum and maximum cell coordinates. These distributions were obtained by repeating the same simulation 300 times with the parameters of Fig. 2 (out of which 30 failed on average and were discarded), only changing the random seed every time. The profiles are essentially bell-shaped, with low variance, showing that our approach based on morphogenetic fields is sufficiently robust to support the morphogenetic engineering of targeted shapes. (B) Morphological characteristics as function of the survival threshold $\theta_{\beta}$, varied in $[0.2,20]$ by increments of 0.2 (all other parameters as in Fig. 2). Since $\theta_{\beta}$ induces death in the POP2 cells (cyan bacteria) which make up the crown, then the lower $\theta_{\beta}$, the larger the crown thickness (cyan curve) and outer radius (black curve) because the inner radius is not affected (yellow curve). All radial sizes were measured directly in the Gro environment based on minimum and maximum cell coordinates. The gray area indicates the standard deviation of the total radius, calculated over 100 simulations in each point (10\% discarded on average). (a-d) Four examples of wheel shapes obtained under different $\theta_{\beta}$ values (final snapshots at homeostatic equilibrium corresponding to $t_{8}$ in Fig. 2), indicated by pins on the horizontal axis. (C) Influence of the diffusion rate $\kappa_{\text {diff }}(\alpha)$ varied in $[0.03,3.0]$ by increments of 0.03 . Since chemical signal $\alpha$ is emitted by the LEADER cell and affects the differentiation boundary between the POP1 and POP2 layers, then the higher its diffusion rate, the wider the inner radius and the total radius while the crown thickness remains constant. (a-d) Four final snapshots of wheel shapes at different rate values. 
for 300 trials under the parametric conditions of Fig. 2. Only about $10 \%$ of the simulations were rejected because two or more LEADER cells emerged and compromised the development of the core. The narrow bell-curve aspect of these distributions shows that our simulated developmental process produces robust morphologies, and legitimates the study of morphological variations through numerical experiments.

In sum, these results highlight the reliability of the morphogenetic engineering approach, based here on chemical fields. The whole assembly is capable of maintaining a wheel shape of a certain size at the same time all its cells (except the leader) are constantly renewed. On the other hand, this happens only as long as there exists a LEADER cell that emits the proper morphogen. Naturally, the dependency on a singularity of this kind is a much less desirable feature since, as soon as the leader dies, the bilayered-core organization disappears through uncontrolled proliferation, then massive death of POP1 and POP2 cells deprived of $\alpha$ and $\beta$ morphogens. Additional mechanisms are required for a more realistic biological implementation of this scenario, either by triggering the spontaneous replacement of the leader (e.g. through self-election) or by allowing a clump of multiple LEADER cells, or by dispensing with the need of leaders entirely (see "Discussion" for a review of alternatives).

MorphoBrick-1 Genomic Variations: Transition Thresholds. We now analyze the influence of the variation of thresholds $\theta_{\alpha}$ and $\theta_{\beta}$, starting with the latter. One of the roles of the survival threshold is to induce death in POP2 cells when they detect a low $\beta$ concentration. Therefore, $\theta_{\beta}$ should have a direct influence on the thickness of the POP2 crown. This is verified in the statistical analysis of Fig. 3B: increasing $\theta_{\beta}$ results in an average decrease of the crown thickness. On the other hand, the inner radius of the POP1 layer remains constant, therefore the outer radius of the whole assembly, which is the sum of both, also decreases. These curves were obtained by simulating 100 specimens for each one of 100 different $\theta_{\beta}$ values, with the other parameters set as in Fig. 2. Note that the thickness decrease is not linear, since at high survival thresholds the border cells are in a steep region of $[\beta]$ gradient, therefore threshold variations incur only relatively small 
thickness variations; whereas at low survival threshold the border cells are in the flatter tail region of the gradient, hence slight threshold variations can cause larger size differences.

A similar analysis was conducted over the differentiation threshold $\theta_{\alpha}$, which controls the POP1 $\leftrightarrow$ POP2 reversible transition. In this case, as expected, the effect was a decrease of the inner radius (hence the total radius) with higher values of $\theta_{\alpha}$ (curve not shown). Unlike the previous scenario, however, $\theta_{\alpha}$ variations end up affecting both geometric measures, not just one of them. When the inner radius becomes too small, the outer crown also collapses, since there are not enough POP1 cells to create a morphogenetic field of $\beta$ suitable to POP2 cells for their development.

MorphoBrick-1 Environmental Variations: Chemical Rates. Phenotypic variations can also be elicited by modifying the properties of the surrounding chemicals, instead of the bacteria. This is a case of "polyphenism", where the same genotype immersed in different environments can produce a different phenotypes. Here, by varying the diffusion and degradation rates $\kappa_{\text {diff }}$ and $\kappa_{\mathrm{deg}}$, the main homeostasis property of the core shape can be preserved while its morphological characteristics are modified. Fig. 3C displays the results of a statistical analysis over a range of diffusion rates of morphogen $\alpha$. As before, 100 simulations were carried out in each one of these 100 points. Unsurprisingly, the curve shows that when the diffusion rate increases, the inner radius and total radius also increase.

We also analyzed the organism size as a function of the degradation rate $\kappa_{\mathrm{deg}}(\alpha)$, which describes how fast morphogen $\alpha$ disappears. In this case, also reassuringly, the higher the degradation rate, the smaller the inner radius (curve not shown). When the degradation rate becomes too high, $[\alpha]$ drops faster than it can be replenished by the LEADER cell's signal emission. This results in a small area of concentration around the leader, hence a small inner layer, which in turn has a damaging influence on the crown thickness as explained above.

MorphoBrick-1 Genomic Mutation: Number of Types. In a last part of this MorphoBrick-1 study, we look at a hand-mutated genome structure, in which a third cell type POP3 is created and new conditional transitions introduced between POP2 and DEAD (Fig. 4A; see full program 


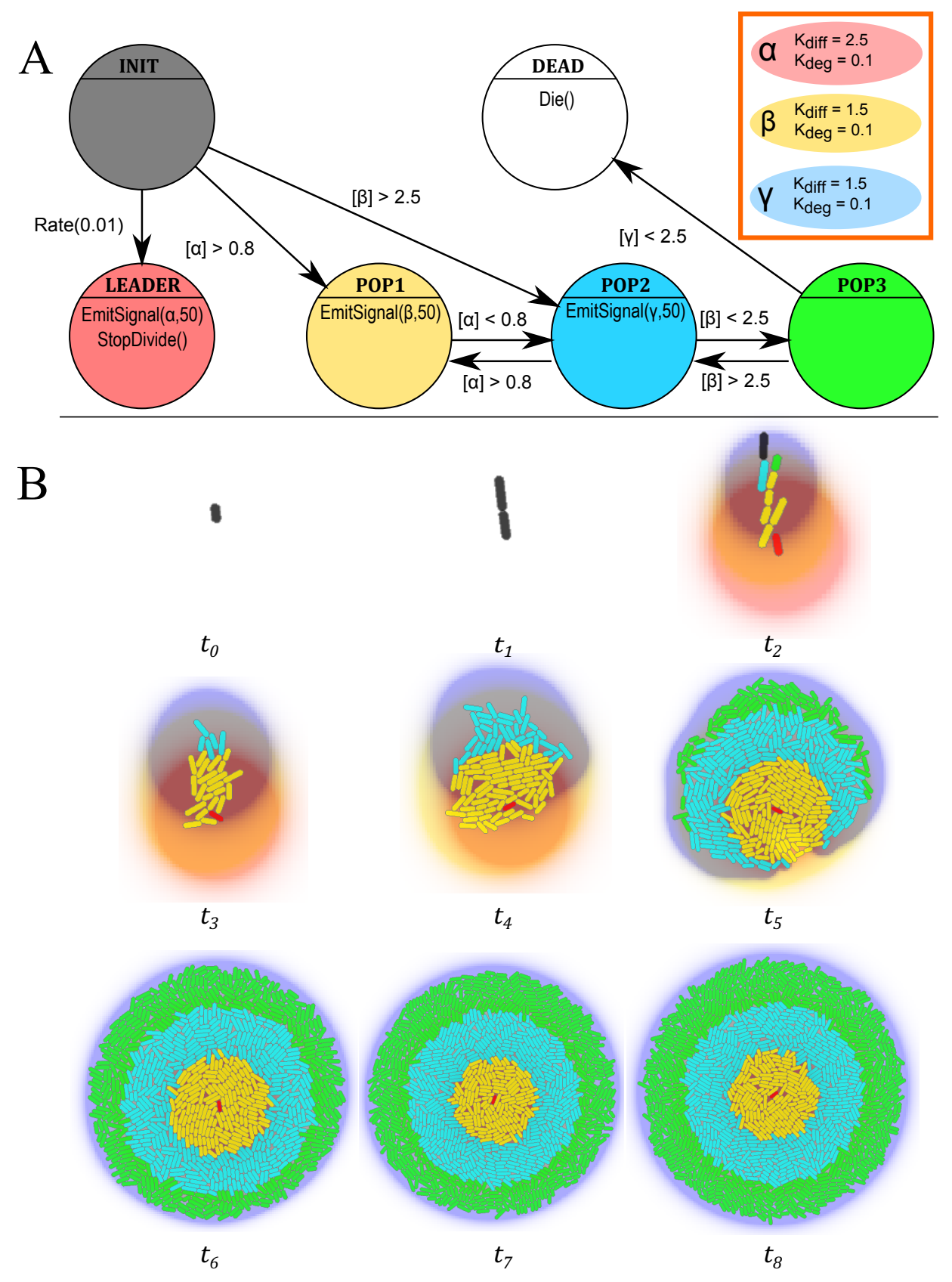

Figure 4. Extra type and chemical in the implementation of MorphoBrick 1, "homeostatic core" (Supporting Information, Video 1b). (A) Compared to Fig. 2, this new genome design contains a third type, POP3, inserted in POP2's path to cell death. Instead of dying at the edge of the $\beta$ gradient, the POP2 bacteria (in yellow) first differentiate into POP3 (in green), then disappear at the edge of a new $\gamma$ gradient emitted by POP2 (with threshold $\theta_{\gamma}=2.5$ ). (B) As a consequence, the growing organism gains a third layer of cells. Notice the spontaneous spatial rearrangement in the last stages of the homeostatic convergence (from $t_{6}$ to $t_{8}$ ), as the initially asymmetric layer disposition caused by the linear, upward growth of populations 1,2 and 3 (from $t_{2}$ to $t_{5}$ ) becomes centered again. 
in Supporting Information, Code 1b). Moreover, POP2 now emits another morphogen, $\gamma$, which sustains the emergence of POP3 cells and also regulates their disappearance on the outer rim. In essence, the INIT $\rightarrow$ POP1 $\rightarrow$ POP2 differentiation chain is augmented with a third link, POP1 $\rightarrow$ $\mathrm{POP} 3$, and the consequence is the appearance of a third layer in the collective phenotype (in green in Fig. 4B). As before, the thickness of the layers and the global radius of the wheel-shaped assembly are parametrized by the various threshold and rate values. Theoretically, this approach can be easily generalized to $N$ POP types: given a desired number of layers $N$, a generative approach could automatically produce the corresponding SBGP genome, then the Gro script.

The advantage of increasing the number of types and layers is to improve positional information, hence positioning diversity and accuracy for the future appendages in later stages (see "MorphoBrick 3" paragraphs below). This is because more signals can be interpreted more locally over shorter distances, where gradients are steeper and less sensitive to noise, i.e. can provide better control than flat ones. On the other hand, the problem is the complexity of handling multiple chemical signals and the difficulty of implementing them in the lab (see "Discussion").

Limb Growth: MorphoBrick 2. The second component of our morphogenetic experiments is a stick, or "limb". Here we describe an implementation of standalone limb development in the multicellular medium. In the next section, we show how it can grow out of the body, using MorphoBrick-3 precursor cells as "joint" structures. The limb's differentiation graph is based on four active cell types: LEADER, QUIESCENT, FLESH and SKIN (Fig. 5; see full program in Supporting Information, Code 2a). Initially, only initial cells of the first two types are needed to start the process, which unfolds in a way similar to meristem offshoot in plants. The LEADER plays the role of a precursor cell, while the QUIESCENT cell, upon stimulation by the leader cell's signal $\alpha$, turns into a FLESH cell and enters a proliferative cycle. Overall, the net effect is that the leader is continually pushed away, leaving a diffusive "plume" of cells in its path. FLESH cells spread out in the region $[\alpha]>\theta_{\alpha}=0.8$ and also emit $\beta$, surrounding themselves with an outer layer of SKIN cells (in the same way POP1 cells surround themselves with POP2 in MorphoBrick 1), to the extent 


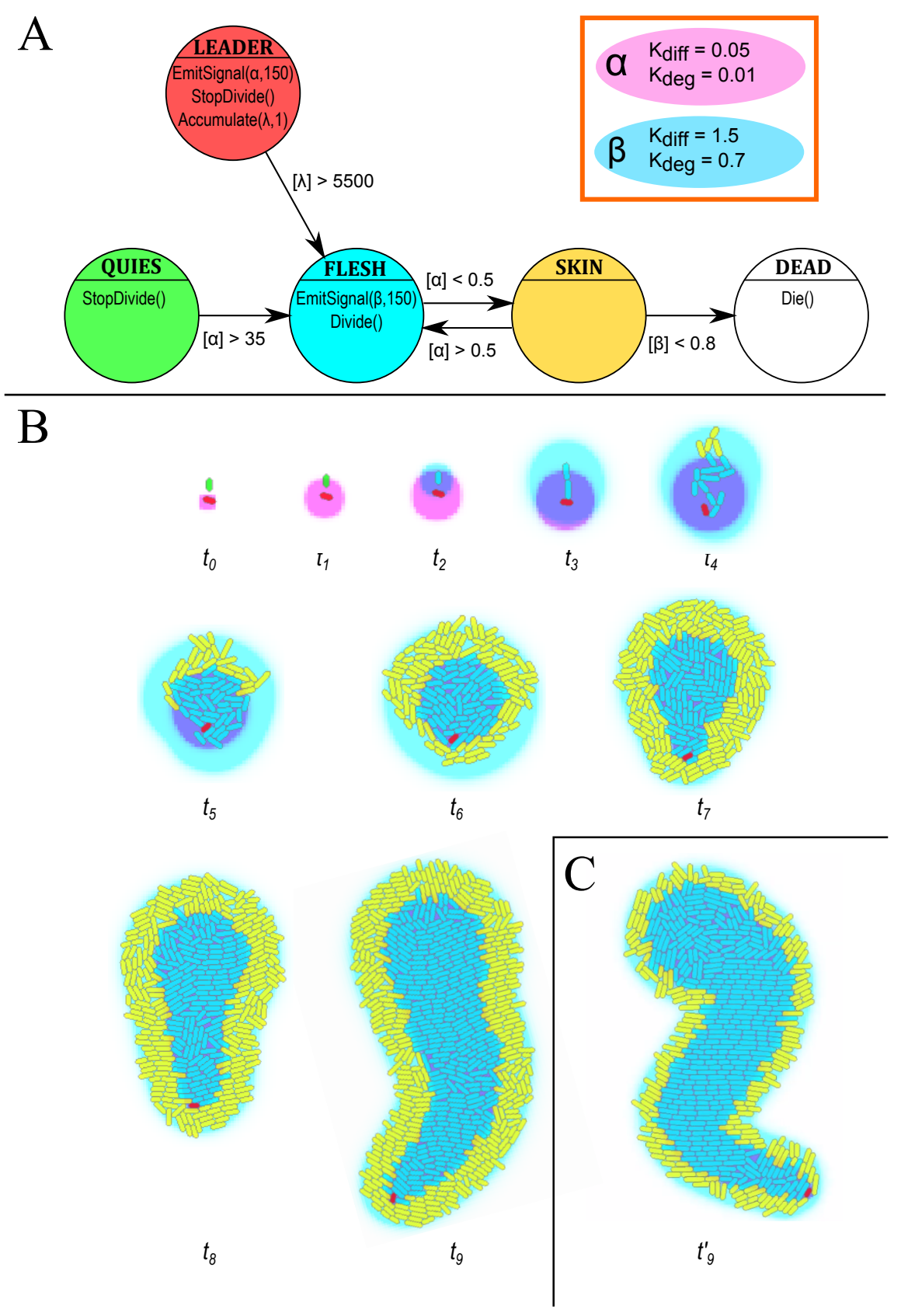

Figure 5. Genomic representation and simulation of MorphoBrick 2, "limb growth". (A-B) Two cells of two distinct types initiate the developmental process: a LEADER cell (in red) and a QUIESCENT cell (in green). The former emits a chemical $\alpha$ activating the latter's proliferation via its differentiation into another type, FLESH (in cyan), which in turn emits a signal $\beta$ where a fourth type, SKIN (in yellow) survives. Similar to MorphoBrick 1 (Fig. 2), the size of the inner and outer regions and yellow areas are parametrized by thresholds $\theta_{\alpha}=0.5$ and $\theta_{\beta}=0.8$. Proliferation and mechanical forces continually push the LEADER cell to the outer skirts of the assembly. Because this cell leaves a trail of $\alpha$ morphogen along its displacement, the result is the development of a "teardrop"-shaped limb (Supporting Information, Video 2a). The final length is determined by the accumulation of an internal protein $\lambda$ in the leader, which differentiates into FLESH beyond a third threshold $\theta_{\lambda}$. (C) Different outcome based on a higher survival threshold $\theta_{\beta}=1.5$, and showing distortions due to a windier path taken by the leader (Supporting Information, Video $2 b$ ). 
determined by a lower threshold: $[\beta]>\theta_{\beta}=0.8$.

The slow diffusion $\left(\kappa_{\text {diff }}=0.05\right)$ and low degradation rate $\left(\kappa_{\mathrm{deg}}=0.01\right)$ of morphogen $\alpha$ emitted by the LEADER cell builds a kind of "chemical shelter" for FLESH cells, as it allows the concentration to stay high and FLESH cells to proliferate while the source cell has time to move away, which fuels the lengthening and widening of the limb. In fact, this is the most important difference with the development of the core (for which $\kappa_{\text {diff }}=2.5$ and $\kappa_{\text {deg }}=0.1$ ). As in MorphoBrick 1, however, the success of limb development rests upon the survival of a single LEADER cell. Here its unicity is guaranteed since the leader is already present at the start and does not need a rare stochastic event to appear later in the process (it will be in fact provided by MorphoBrick 3 in the next stage). Yet again, the same questions as before arise (see "Discussion"): can the design be modified to accommodate the regeneration of the LEADER cell in case of its untimely disappearance? Or could there be a clump of multiple leaders—or none at all?

In any case, a specificity of the limb structure is that its extension depends on the life duration of the LEADER cell. Here homeostatis is only partial, in the sense that the SKIN layer adopts a certain thickness while still proliferating, whereas no limit length is reached and maintained. Therefore, to control the length of the limb, we introduce a clock mechanism in the source. Once it starts emitting the proliferation activator $\alpha$, it also accumulates an internal protein $\lambda$, and when this concentration reaches a given threshold $\theta_{\lambda}$, then LEADER differentiates into FLESH, mingles with the crowd, and the growth process stops. The limb length can therefore be parametrized in two ways: by varying $\theta_{\lambda}$ or the accumulation rate $C_{\lambda}$ of the protein. The sensitivity to noise and the influence of the genomic and chemical parameters on the geometrical features are studied next.

MorphoBrick-2 Sensitivity Analysis. As in the previous section, it is important to analyze the robustness of limb growth, if we want to use it as a reliable developmental building block. To this aim, we performed again 100 runs under the default parametric conditions of Fig. 5 and measured the average thickness and length of the obtained morphology (Fig. 6). Both were obtained by extracting the topological skeleton from the limb shape, i.e. the pixelized domain covered by 

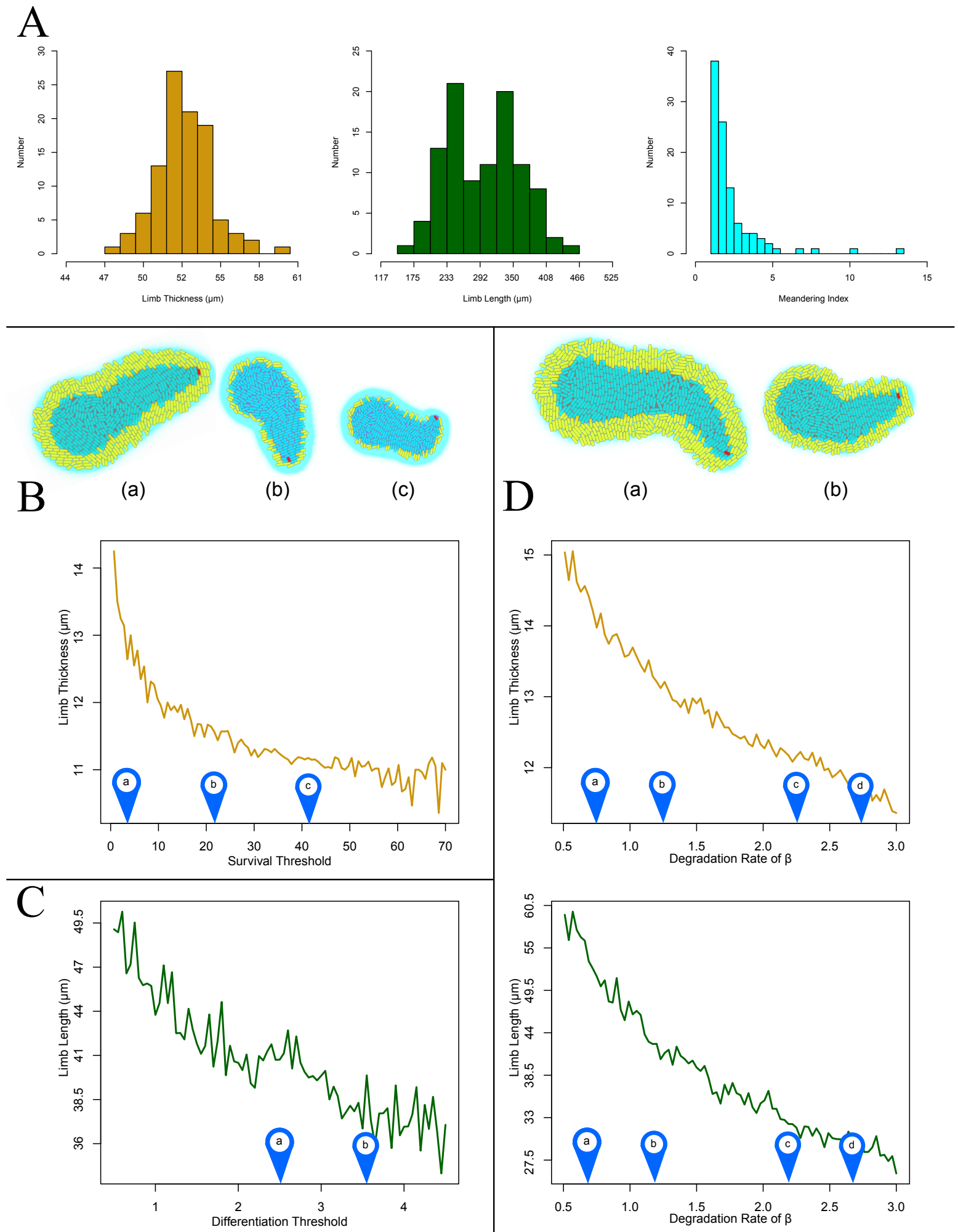

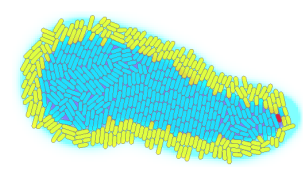

(a)

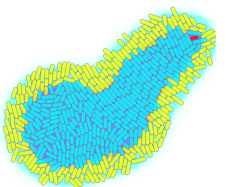

(b)
D
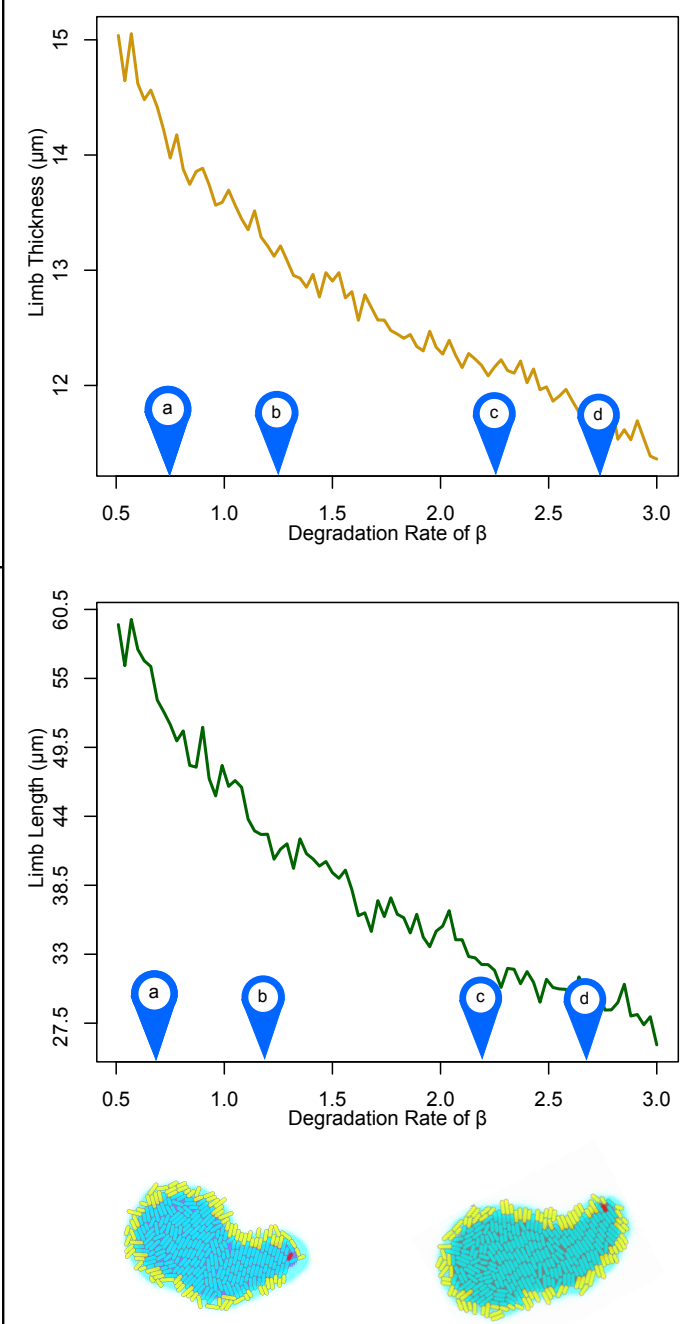

(c)

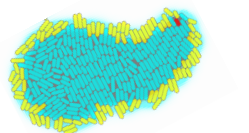

(d) 
the bacteria after smoothing to avoid too many spurious branches (see algorithm in Supporting Information, "Morphological Analysis"). The thickness corresponds to the number of iterations required to "eat out" the flesh and converge to the skeleton, while the path length is given roughly by the number of pixels that constitute the final skeleton (see conversion to $\mu \mathrm{m}$ in figure caption). As with the homeostatic core structure, these distributions appear essentially bell-shaped with a relatively narrow variance, except for a slight tendency of the length distribution to bimodality, showing a relative lack of mid-range values. A third measure, the meandering index, which is the limb length divided by distance between the initial and final positions of the LEADER cell, indicates that the great majority of phenotypes are linear (value close to 1) —although a significant number of them are also sufficiently curved or convoluted to produce indices of 2 or 3 (e.g. hook-shaped limbs whose extremities are close to each other).

MorphoBrick-2 Genomic Variations: Transition Thresholds. Next, we need again to understand the effect of genomic parameters and environmental conditions on the phenotype to be able to target specific morphological characteristics. Since morphogens $\alpha$ and $\beta$ play a role equivalent to the MorphoBrick-1 study, we also call $\theta_{\alpha}$ "differentiation threshold" and $\theta_{\beta}$ "survival threshold"

Figure 6 (preceding page). Sensitivity analysis and parametric exploration of MorphoBrick 2, "limb growth". (A) Distributions of three geometric measures, from left to right: limb thickness, limb length, and meandering index. The limb thickness corresponds to twice the number of iterations required to finish skeletonization, where each iteration removes a 1-pixel thick layer, then applying a rescaling factor of 10 pixels $=5.8 \mu \mathrm{m}$ (without correcting for the possible excess of pixels in diagonal "staircase" segments, compared to straight segments). Through morphological analysis, the limb length corresponds to the number of pixels remaining after skeletonization, applying the same scale. The meandering index is a unitless quantity equal to the limb length divided by distance between the leader's initial and final positions. These distributions were obtained by repeating the same simulation 100 times with the parameters of Fig. 5, only changing the random generator seed every time. As in Fig. 3A, the first two distributions are bell-shaped, except for some bimodality in the length feature. The third one shows that most limbs grow out straight, a few others end up curved, with extremities very close. (B) Organism thickness as a function of survival threshold $\theta_{\beta}$. (C) Organism length as a function of differentiation threshold $\theta_{\alpha}$. (D) Thickness and length as a function of degradation rate $\kappa_{\operatorname{deg}}(\beta)$, varied in $[0.03,3]$ by increments of 0.03 (other parameters as in Fig. 5). All sizes calculated by morphological analysis, here with a rescaling factor of 10 pixels $=2.7 \mu \mathrm{m}$. In each case, means are calculated over 100 simulations for each parameter set, and images (a) to (d) show examples of shapes obtained under different values just before final differentiation of the LEADER cell (in red) and growth stop (equivalent to $t_{9}$ and $t_{9}^{\prime}$ in Fig. 5), indicated by pins on the horizontal axis. 
here. For reasons similar to the previous study, the results shown in Fig. 6B,C confirm that an increase in $\theta_{\beta}$ provokes a decrease in the thickness of the SKIN layer (formerly POP2), hence the whole limb, while an increase in $\theta_{\alpha}$ provokes a decrease in the FLESH population size (formerly POP1), hence the overall length since the leader is pushed away slower by less active proliferation and packing forces.

MorphoBrick-2 Environmental Variations: Chemical Rates. External conditions also influence the developmental process. Here we display the influence of the degradation rate $\kappa_{\mathrm{deg}}$ of the survival morphogen $\beta$ (Fig. 6D). A higher rate causes a decrease in both dimensions of the limb, thickness and length, although in different ways. Clearly, the more degradable $\beta$, the more restricted the area where $[\beta]>\theta_{\beta}$, hence the less prolific the SKIN cells. If $\kappa_{\mathrm{deg}}$ is too high, the morphogen never reaches a level suitable for SKIN to develop and survive. The FLESH cells, for their part, are not sensitive to $\beta$, yet limb length still indirectly depends on the degradation rate. The reason, again, is that the trajectory and speed of displacement of the LEADER cell along the major axis are a result of mechanical interactions and spatial constraints exerted by the SKIN layer pressing laterally on the FLESH core. In the case of a more volatile chemical, there is less density and these forces are weaker. Note that, in any case, cleavage planes are oriented randomly and do not contribute to the direction taken by the elongation; only the leader's displacement does.

Precursor Cells and Assembly: MorphoBrick 3. The third and last component we present in this study consists of placing at the periphery of a wheel a precursor cell that will give rise to a stick. For this, we need a spatiotemporal differentiation mechanism by which certain POP2 cells around the core can self-elect at regular intervals and become the new LEADER cells of limb growth. This is a form of segmentation, a pervasive developmental episode across multicellular species (e.g. somitogenesis in vertebrates), which will be based here on the combination of two low-level mechanisms: wave propagation and oscillations. 

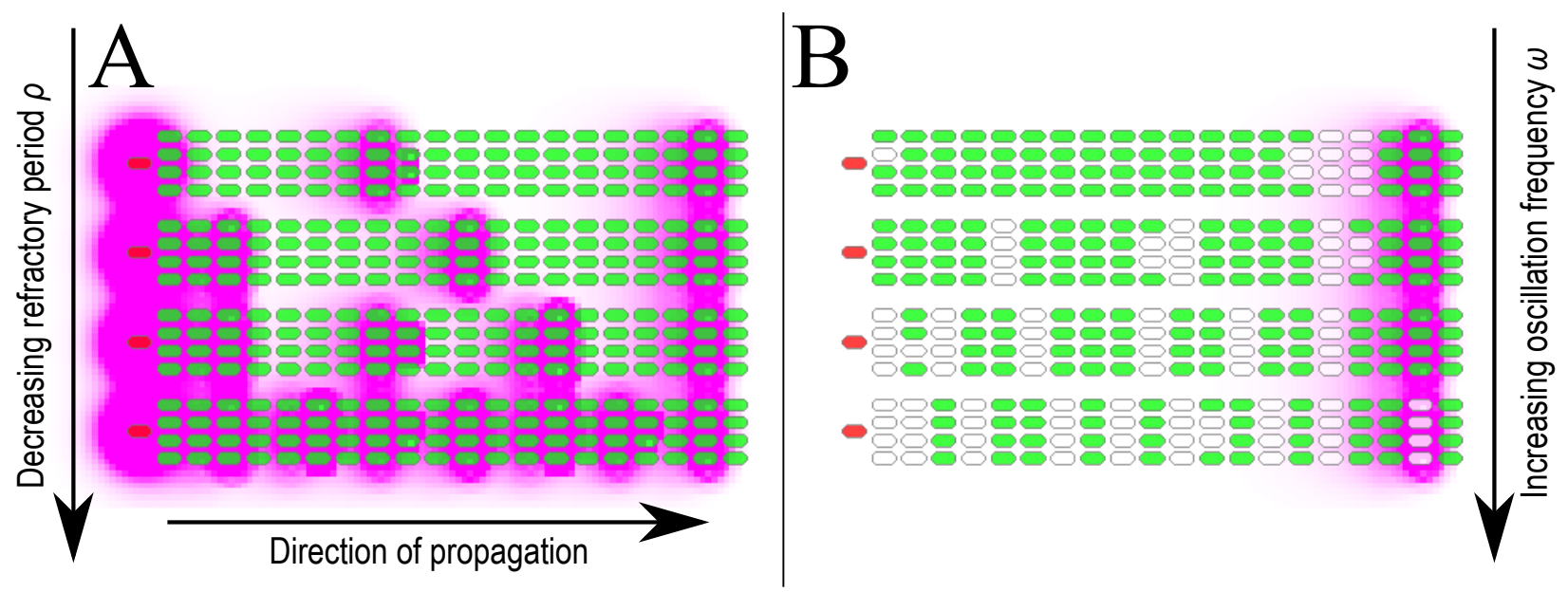

Figure 7. Segmentation mechanism at the basis of MorphoBrick 3, "precursor cell positioning". The directed propagation of a wave of morphogen (pink cloud) across a fixed band of $4 \times 20$ cells (here displayed four times) results from their detection-and-reemission behavior interrupted by a refractory period $\rho$ (i.e. they behave like "excitable units"). It is combined with an oscillatory state of angular frequency $\omega$ in each cell to produce a striped pattern of differentiation (white type), which will be the site of precursor cells (Supporting Information, Video 3c). (A) Decreasing refractory periods under $\omega=2.5$ rad. $\mathrm{min}^{-1}$ (i.e. an oscillation period of $T=2 \pi / 2.5=2.5 \mathrm{~min}=25 \Delta t$ ), from top row to bottom row: $\rho=8,6,4,2 \mathrm{~min}$. (B) Increasing oscillation frequencies under $\rho=6 \mathrm{~min}$, from top row to bottom row: $\omega=0.5,1.5,2.5,3.5$ rad. $\mathrm{min}^{-1}$ (i.e. oscillation periods $T=12.6,4.2,2.5,1.8 \mathrm{~min}$, respectively).

MorphoBrick-3 Segmentation. Wave propagation is generated by signal emission and detection via a refractory period $\rho$ in each cell. It is illustrated in Fig. 7A on a fixed rectangular grid of cells. A signal (pink cloud) is emitted once by a special cell (in red) at one end of the chain. When neighboring cells are stimulated by this signal, they produce it in turn. After emission, however, each cell waits for a small period of time before it is able to detect-and-emit again. In this manner, the morphogen can be transmitted only in one direction (here from the left to the right). This phenomenon is typical of "excitable media", such as the Belousov-Zhabotinsky reaction or cAMP communication among slime mold amoebae. Clearly, the longer the refractory period, the longer the wavelength.

By coupling this propagation mechanism with an internal clock in each cell, one can obtain a segmentation of the grid into alternating stripes representing two cell types (Fig. 7B): a differentiated type (in white), which appears when the wave traverses the cell at the same time that its internal phase reaches a certain value (say, $2 \pi n$ ); and an undifferentiated type (in green) if wave 
passage and clock phase do not coincide. The number of segments and their typical widths depend on the refractory period $\rho$ and the oscillation frequency $\omega$, in addition to several other factors such as the chemical rates $\kappa_{\text {diff }}$ and $\kappa_{\text {deg }}$, and the phase window $\Delta \phi$ of differentiation opportunity around $2 \pi n$.

MorphoBrick-3 Precursor Cell Positioning. The emergence of segments is only the first stage of the precursor cell positioning process. The differentiated stripes constitute groups of cells eligible to become leaders of a limb growth, but in the current state of the model there can be only one such cell per group. Therefore, another rare stochastic event is needed to allow one candidate cell to self-elect and quickly shut down its neighbors via a local inhibitor signal. Both stages largely overlap: the reduction of groups of candidates to single leaders may already happen while the wave is still running and the segmentation process has not finished creating all the groups.

Such a scenario is illustrated in Fig. 8A (see full program in Supporting Information, Code 3a): first, at $t_{9}$, a signal is triggered from a random seed cell and sweeps across the circular layer of POP2 cells (here in yellow) in the same way as the linear band of Fig. 7 (on immobilized bacteria, to simplify the experiment). The difference here is that the wave propagates symmetrically in both directions (the signaling morphogen is not shown), and the formerly undifferentiated cells are now converted into a "disabled" black type when traversed by the wave outside of their phase window. Then, at $t_{12}$ one of the candidate white cells from the group in the lower left quadrant differentiates into a limb LEADER and emits another short-range morphogen (pink clouds), which has the effect of deactivating the other white cells from this group (making them black). Meanwhile, the wave has finished covering the whole outer layer at $t_{14}$ : in this case, the $\rho$ and $\omega$ values are such that a total of five radial stripes (white groups) are created. Short thereafter, the other four groups reduce themselves to single LEADER cells, too (in red). The result at $t_{17}$ is an approximately regular pentagonal structure. In this case, the final layout of the five precursors will always be a pentagon; only its absolute orientation (between 0 and 72 degrees) may vary depending on the location of the first seed cell. This is not a problem for Classes I to III, but in Classes IV and V additional 

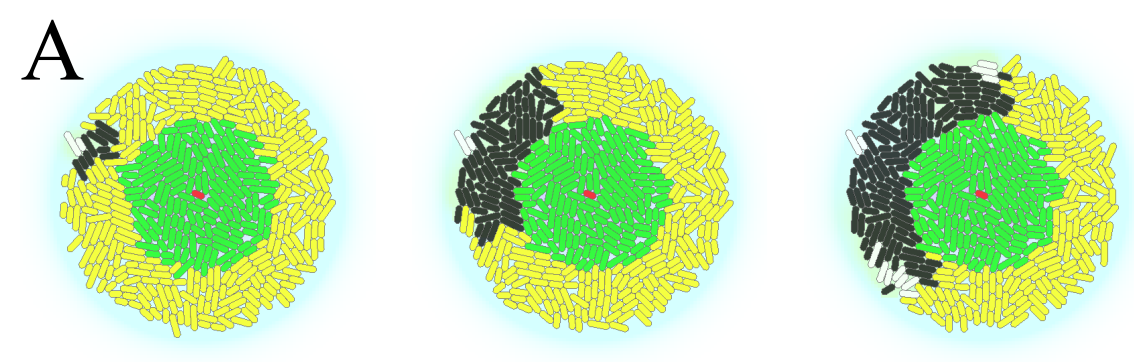

$t_{9}$

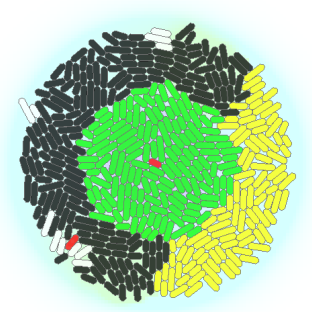

$t_{10}$
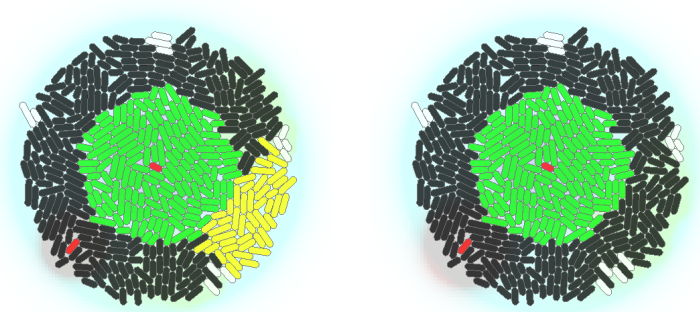

$t_{12}$

$t_{13}$

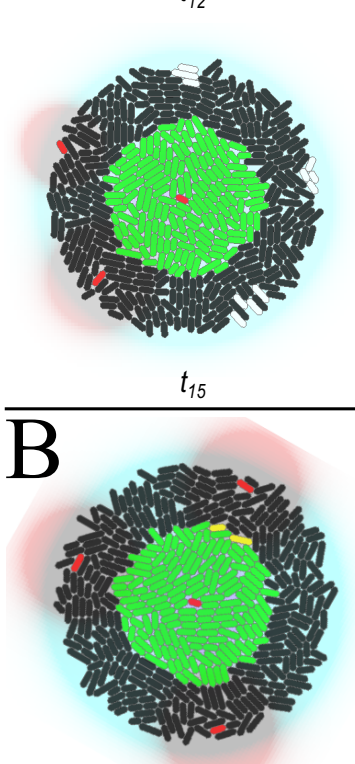

(a) $t_{17}, \omega=0.9$
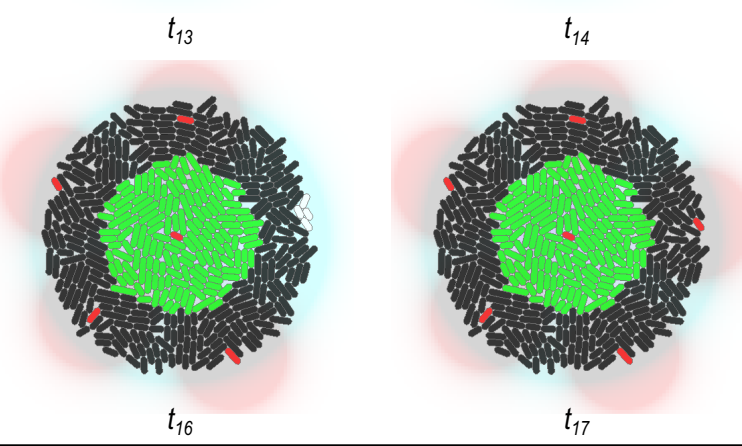

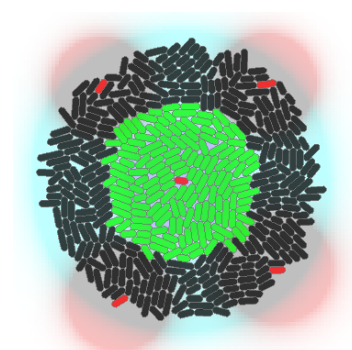

(b) $t_{17}, w=1.25$

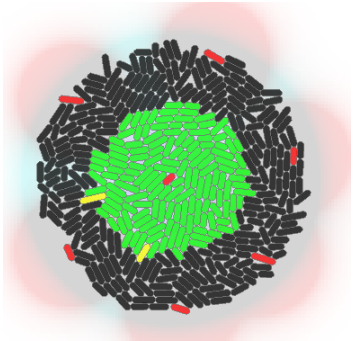

(c) $t_{17}, w=1.88$

Figure 8. MorphoBrick 3, "precursor cell positioning". Development starts from MorphoBrick 1 at $t_{8}$ (Fig. 2) with different colors (POP1 in green, POP2 in yellow) and parameter values $\rho=8$ min, $\omega=1.4$ rad. $\mathrm{min}^{-1}$ (i.e. $T=2 \pi / 1.4=4.5 \mathrm{~min}$ ). To simplify, bacteria are also artificially held in place. (A) $\left(t_{9}-t_{14}\right)$ The first stage consists of a double wavefront on the outer POP2 layer, clockwise and counterclockwise, establishing five groups of candidate precursor cells (in white) and deactivating all the others (in black) in the same way as Fig. 7. $\left(t_{12}-t_{17}\right)$ Largely overlapping, the second stage consists of the spontaneous reduction of each group of candidate precursor cells into single LEADER cells (in red) by stochastic selfelection and quick snuffing of the neighbors (from white to black), based on a short-range inhibitor signal (red cloud). The outcome here is the basis for a five-pointed star shape (Supporting Information, Video 3a). (B) Different internal oscillation frequencies $\omega$ give rise to different degrees of periodicity around the core, e.g. three, four or six-pointed stars (respective oscillation periods: $T=7,5,3.3$ ). In some cases, a few POP2 cells (at the border with POP1) have escaped the inital wave of deactivation, but their spontaneous differentiation into new candidate cells remains extremely unlikely. 
staging mechanisms (possibly involving external stimuli such as light) must be introduced to align or correlate limbs between distant bodies. Fig. 8B shows different degrees of periodicity (triangle, square, and hexagon) arising from different oscillation frequencies $\omega$.

Class-I Shapes: Radially Symmetric Wheels. Putting all three types of MorphoBricks together, we can create the first shapes belonging to Class I, composed of a wheel with a radially symmetric arrangement of limbs. Once the core has developed (MorphoBrick 1) and the precursor cells have appeared (MorphoBrick 3), local proliferation is stimulated at the root of the limbs, provoking their growth (MorphoBrick 2). This latter part is shown in Fig. 9A (see full program in Supporting Information, Code 4a): starting from the last step of Fig. 8, we arrive at a four-armed starfish organism. Fig. 9B shows the variants originating from three, five and six precursor cells.

MorphoBrick-3 Precursor Cell Differentiation. Modularity is a fundamental principle of genotypephenotype economics in development and evolution (44). Biological organisms often contain numerous repeated or serially homologous parts in their body plan (45). This is most striking in the segments of arthropods (several hundreds in millipedes) or the vertebrae, teeth and digits of vertebrates. After duplication, however, these parts tend to diversify and evolve more specialized structures (lumbar vs. cervical vertebrae, canines vs. molars). Homology exists not only within individuals but also between different species, as classically shown by comparing the forelimbs of tetrapods from the bat to the whale. Recently, genetic sequencing has revealed that many stretches of DNA are in fact identical or highly similar. This came in support of the idea that homology is the evolutionary result of duplication followed by divergence through mutation-and, sometimes, loss again.

Accordingly, to reach the radially asymmetric shapes of Class II, an additional mechanism of superdifferentiation among precursor cells is needed. In this way, sticks can be activated or inhibited at certain positions around the wheel, and also ascribed different genomic parameters to obtain different geometrical characteristics (here mostly different lengths). This is implemented here on a four-pointed wheel in several steps (Fig. 10; see full program in Supporting Information, 

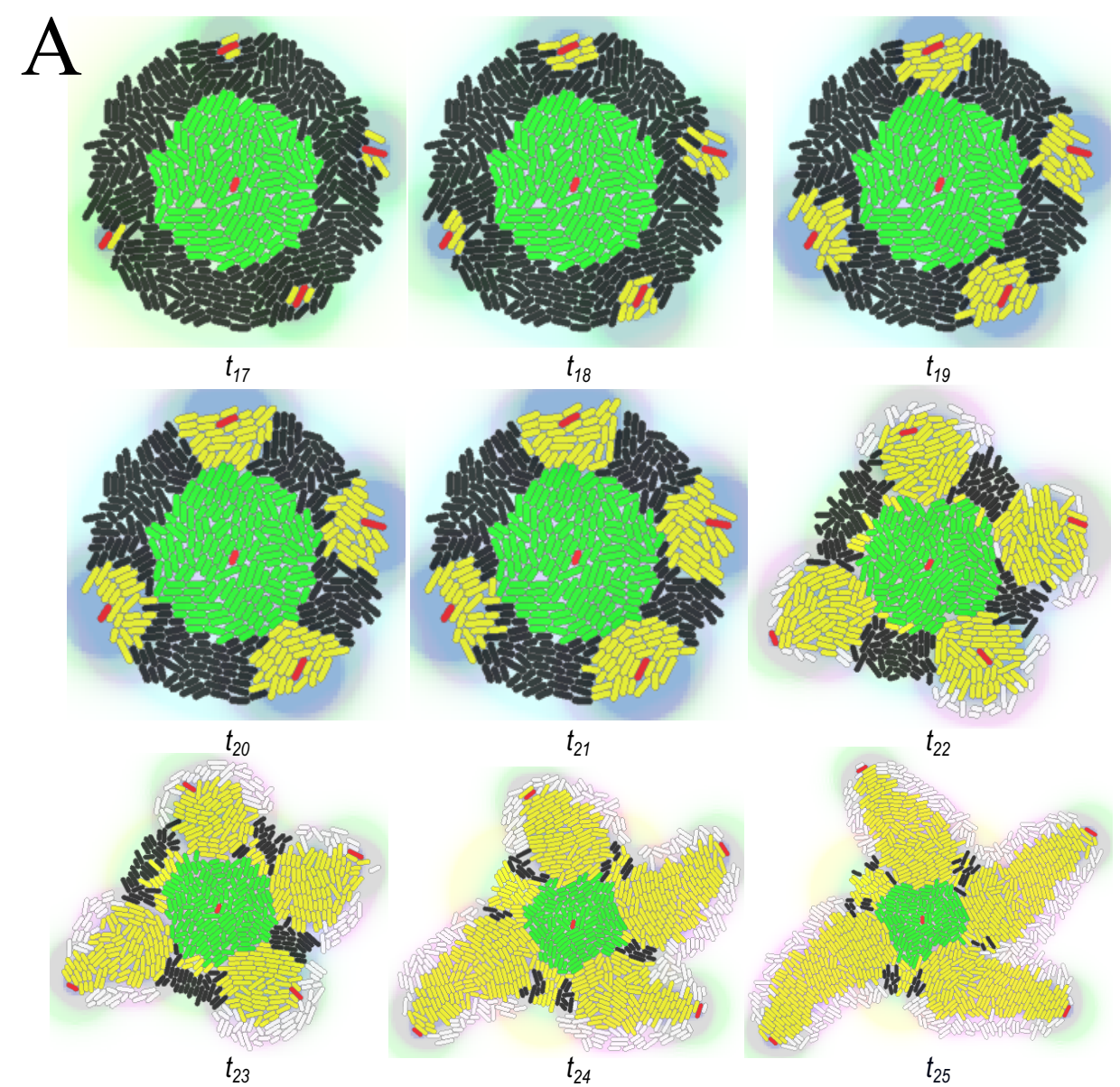

$t_{21}$
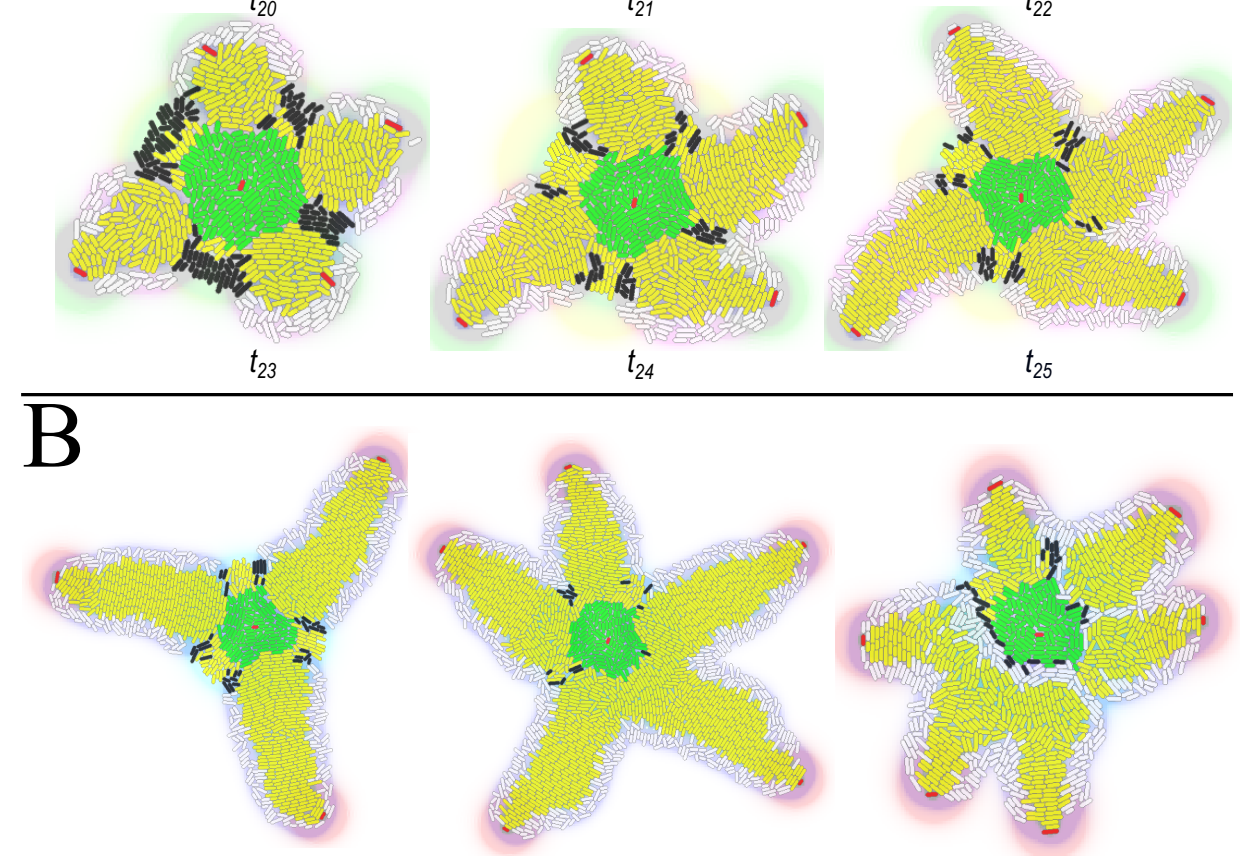

(a) $t_{25}, \omega=0.9$

(b) $t_{25}, w=1.4$

(c) $t_{25}, \omega=1.88$

Figure 9. Class-I shapes: radially symmetric wheels (Supporting Information, Video 4a). (A) A four-armed starfish organism is obtained by chaining the three MorphoBricks presented in the above sections (Fig. 2, 8 and 5) with parameters $\rho=8 \mathrm{~min}, \omega=1.25 \mathrm{rad} \cdot \mathrm{min}^{-1}(T=5)$. Here precursor cells are of the same type and lead to homogeneous limbs (compare with Fig. 11). (B) Different starfish organisms can be obtained by varying the number of precursors (here three, five or six) via the oscillation frequency value $\omega$. 

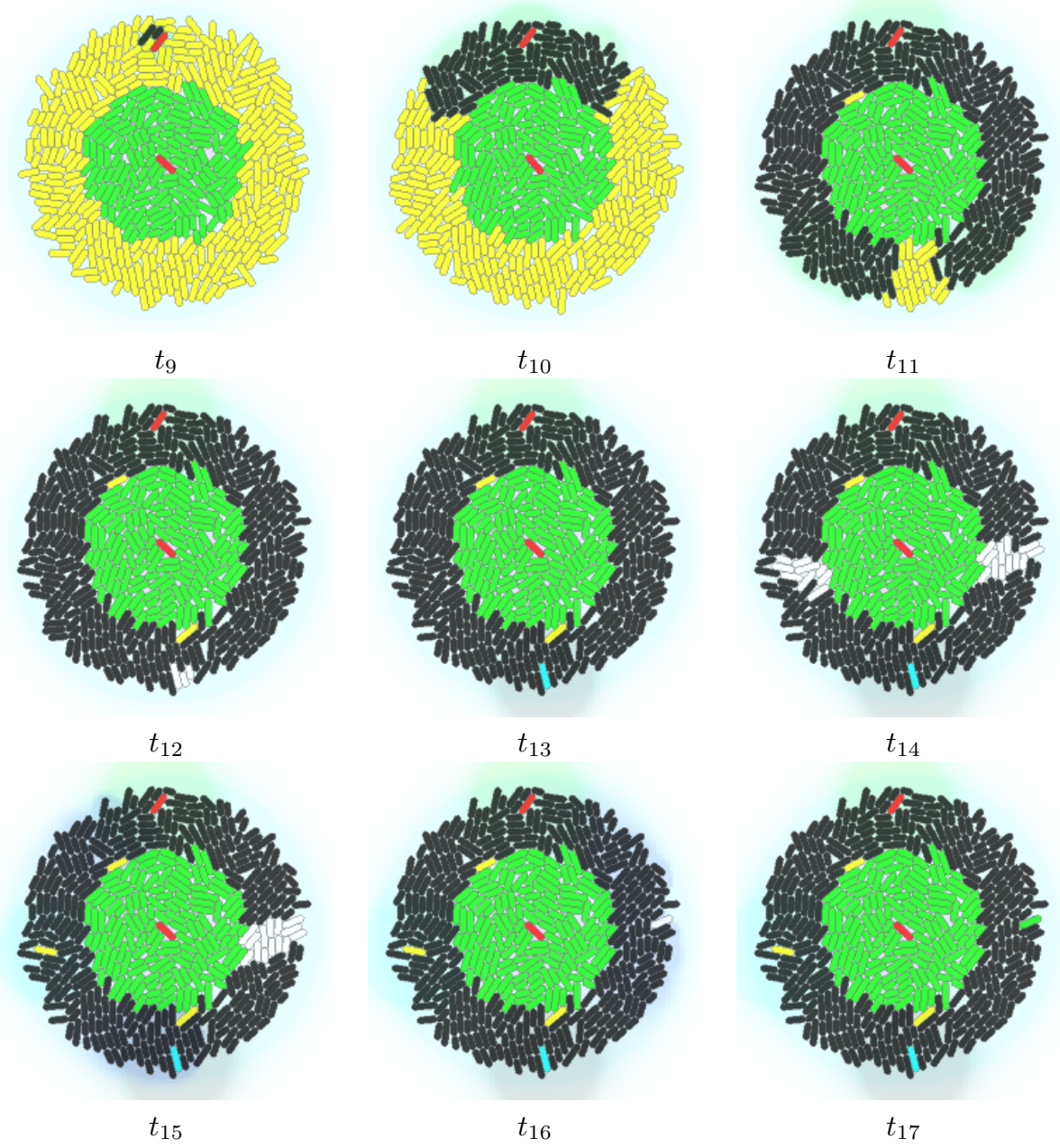

Figure 10. Precursor Cell Differentiation (see text). $\left(t_{9}\right)$ Homeostatic core and emergence of North precursor. $\left(t_{10}-t_{11}\right)$ First wave propagation. $\left(t_{12}-t_{13}\right)$ Emergence of South group and precursor cell. $\left(t_{14}\right)$ Equatorial groups. $\left(t_{15}\right)$ West precursor cell. $\left(t_{16}\right)$ Second wave propagation. $\left(t_{17}\right)$ East and final precursor cell (Supporting Information, Video 3b).

Code 3b): first, with respect to the wave origin (North red cell), $\omega$ is decreased to the point that only one candidate group appears at the antipode, in effect producing a two-pointed wheel $\left(t_{9}-t_{12}\right)$. Then, an essential difference with symmetric organisms is that the spontaneous reduction of this antipode group produces another type for the self-elected leader (South cyan cell, $t_{13}$ ) compared to the inital cell. Next, two new groups emerge on the equatorial line between the first two groups $\left(t_{14}\right)$, but this time based on the MidPlane condition presented in the "Methods" section. Finally, another wave based on a new morphogen propagates out of a self-elected cell from one of the equatorial groups (West yellow cell, $t_{15}$ ) and eventually determines the last precursor (East green 
cell, $\left.t_{17}\right)$ in the same way as the first North/South pair.

After that, it is possible to insert four other specialized points, NW, NE, SW and SE, at $45^{\circ}$ betwen the first four precursors by relying again on the MidPlane condition. In sum, the mechanism creating asymmetrical precursors leads to a four-pointed or eight-pointed wheel (and generally $2^{n}$, if there is enough material and space), since it must start with a pair of antipodes, then another pair of antipodes on the equator of the first pair. It cannot produce an odd number of points. This is hardly a restriction, however, since the goal was precisely to tune each precursor independently from the others so that diverse types of appendages can grow at different angles around the crown. Therefore, multiples of $90^{\circ}, 45^{\circ}$ or even $22.5^{\circ}$ can do as well, if not better, than regular configurations based on $120^{\circ}, 72^{\circ}$ or $60^{\circ}$.

Class-II Shapes: Asymmetric Wheels. At this point, the homeostatic core with asymmetrically arranged precursor spots is akin to an early-stage embryo: no morphology is visible yet, but the "hidden geography" (46) of the body plan is already in place and "divergent homologous" appendages are ready to grow. Fig. 11 presents two experiments producing an L-shape and a T-shape (see full programs in Supporting Information, Code 4b,c): starting from the end of Fig. 10, limbs of different lengths develop from of certain precursor cells elected as limb leaders (North and East for the "L"; West, South and East for the "T") but not others, whose type prevent them from proliferating.

\section{- DISCUSSION}

The ultimate goal of these proof-of-concept numerical experiments in morphogenetic synthetic biology is to help bridge the reality gap toward actual implementation in biosystems. The work presented here should contribute to the automated design and "compilation" of genomes, from high-level scripts such as SBGP and Gro down to DNA sequences, supporting an algorithmic developmental process based on self-made and self-organized components. Strong assumptions had to be made in order to realize the simulations presented here, some of them still relatively far 

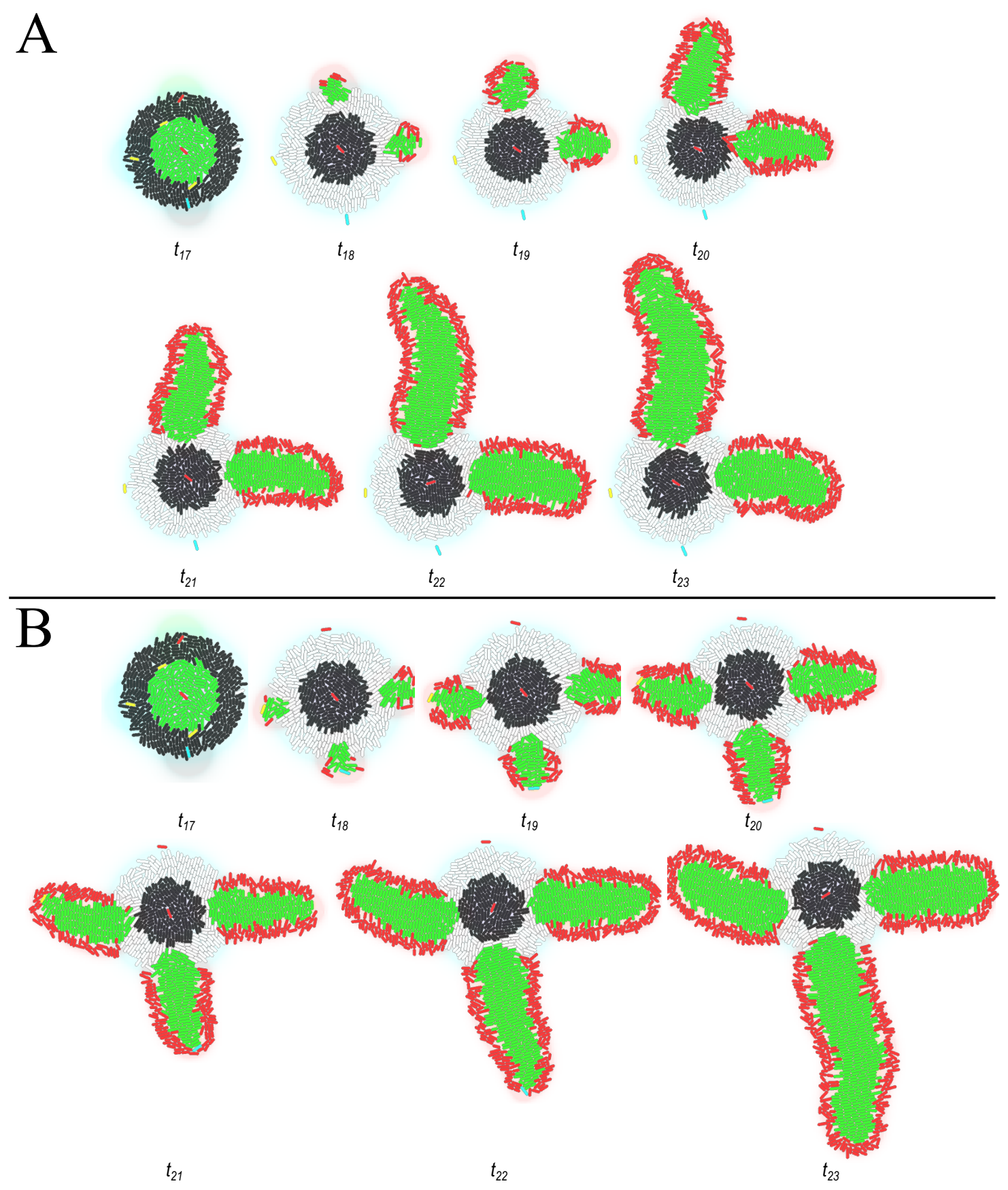

Figure 11. Class-II shapes: asymmetric wheels. (A) Development of an L-shape (Supporting Information, Video 4b). (B) Development of a T-shape (Supporting Information, Video 4c). Note that both organisms start with the exact same core component (MorphoBrick 1) in $t_{17}$ due to an identical random generator seed. Only after that do they diverge because of their genomic differences in precursor differentiation (MorphoBrick 3) and limb growth (MorphoBrick 2). 
from biological plausibility. In this section, we bring elements of answers to the issues raised by our approach. The first point concerns the sustainability of morphologies resting upon single leaders. Then, the multiplicity of diffusive chemical species could also be a problem when envisioning a wet-lab transfer. Finally, we discuss the difficulties of getting a globally synchronized population and the feasibility of the MidPlane construct.

Robustness of Leader Singularity. The main weakness of our approach is that it seems to largely depend on the emergence and survival of a unique precursor or "leader" cell. On the one hand, if multiple MorphoBrick-1 leaders pop up, the homeostatic core will exhibit an irregular form that will perturb the wave propagation and subsequent number of limbs. Moreover, if an emerging MorphoBrick-3 precursor cell cannot inhibit its neighborhood rapidly enough, the resulting limb could split during its growth and lead to an abnormal appendage. On the other hand, if a unique leader disappears for any reason, e.g. external stress, the chemicals that it emits quickly vanish due to degradation, driving surrounding cells to die. Eventually, the whole organism collapses as all the morphogenetic fields that support its structure disappear.

What kind of mechanism could be envisioned to allow leader regeneration and ensure organismal sustainability? For example, a "death alert" signal could be introduced in the leader cell's behavior, e.g. it could emit a specific protein upon transitioning to cell death. Next, cells that are close to the leader (the first layer of the homeostatic core) could try to differentiate and self-elect to the rank of new leaders when they detect this alert protein. Then, the first new leader emits an inhibitor to ensure that it remains unique. Yet again, despite being more adaptive, this approach still relies on the maintenance of a single leader and, even with protection mechanisms in place, any incident during the growth process could lead to an abnormal phenotype. Therefore, it is important to come up with a genuinely decentralized and robust system in order to tackle this problem, either by multiplying leaders or by forgoing the LEADER type altogether.

A well-known behavior evolved by living bacteria to ensure homeostasis of the group is quorum sensing (QS) (47), the ability of individual cells to sense and react to the density of the colony. 
Under stress, bacteria can emit a chemical that triggers cell death above a certain concentration, resulting in self-regulated population size. Within the great diversity of QS signals and behaviors, an example is S. pneumoniae which can differentiate into a "competent" state, i.e. one where the cell is more prone to genetic mutation but also, at the same time, to growth arrest and autolysis based on a QS-dependent on/off switch (48). Thus a possible way to solve the dependency on one or several leader cells would be to use a QS mechanism to limit proliferation. In MorphoBrick 1, starting with all initial cells already in the POP1 type, a proper balance between diffusion, degradation and emission rates could probably be found in order to create a stationary concentration of morphogens, hence a stable POP1 cluster. It is also likely that this cluster would stabilize into a roughly circular shape, hence that the core would correctly develop from there as planned. Alternatively, it should also be possible to reinforce the tendency to form a circle by using a small cluster of LEADER cells in lieu of a single one. Thus rather than installing QS among the POP1 cells, it would be practiced by the LEADER cells and POP1 would then simply grow around them. More layers could also be added (as in Fig. 4) to improve the roundness of the shape even further.

Multiplicity of Chemical Species. Another aspect that could make a direct application to in vivo experiments problematic is the multiplicity of diffusive chemical species hypothesized in the environment. For example, the Class-II organisms presented here (the letter shapes) consume no less than seven different and highly specific signals. At higher levels of biological complexity (Class III and beyond), the number of chemical species involved in bacterial communication will be even greater according to the MorphoBrick compositional design. Moreover, in the current framework, no chemical reactions are modeled, i.e. molecular species are not supposed to react with each other if we want to avoid side-effects during development. These conditions will be a challenge to maintain in the real world. A more realistic model will need to allow reuse of morphogens in different locations and at different stages of the organismal growth. It must also take reaction kinetics into account and exploit the new compounds thus produced to serve as new signaling molecules. On the other hand, the added complexity of a network of chemical reactions will 
also require fine-tuning of a great number of parameters, which can ultimately be only achieved by evolutionary computation (such as genetic algorithms)—or, why not, actual biological evolution, by letting the artificially engineered bacteria re-adapt to new conditions on their own.

Synchrony of Bacterial Population. So far, to ensure the success of MorphoBrick 3, the bacterial population needs to be synchronized. The coordinated polarization of the initial core of the organism is necessary to allow conditional differentiation of candidate precursor cells at regular intervals on the outer crown. Yet, this is the only stage where global synchrony is required: at any other point during development, bacteria's internal clocks or oscillatory behavior are not part of the dynamics. Therefore, it would be more realistic to devise a solution relying on local synchrony only. This said, the method of forcing cell synchronization, then measuring how they gradually get out of phase during relaxation is commonly employed to analyze cell cycles, and in silico modeling and simulation of this phenomenon were also proposed (49). This is why we assumed that the global synchrony stage was a natural and relatively easily reproducible phenomenon, and we could dispense with a more accurate depiction of it in our present numerical framework. At the synthetic GRN level, we can briefly mention the work of Danino et al. (50), who propose engineered genetic circuits able to produce and maintain synchronized oscillations in a growing population of cells. This could for example constitute a suitable component in a future implementation of MorphoBrick 3.

Midplane Feasibility. Finally, growing organisms of Class II and above depends on the MidPlane mechanism, which allows a cell to recognize whether it is approximately midway between two different sources of chemical gradients. In the abstract, this is achieved by comparing local product concentrations and assessing whether they are sufficiently close. In reality, the question of how the required combination of "subtracter" and "zero-tester" logic is implemented in the GRN can be answered by signal-mediated toggle-switch subcircuits. For example, two repressible promoters can be arranged in a mutually inhibitory loop (13) or concurrent promotion/repression can be exerted by the same transcription factor in conjunction with two different signals (51). In any case, 
there are several possibilities of making the regulation dynamics exhibit bistability, such that a cell can fall into one of two states depending on the relative levels of two ligand concentrations it receives.

\section{- FUTURE WORK}

Toward Higher-Level Classes. This paper covered Class-I and Class-II organisms. To reach higher-level classes, other issues need to be solved. First, simulations should be run at a lower computing cost and/or time, since for now almost an entire hour is needed to calculate the development of one T-shape or L-shape (even as a batch job, without online visualization). This could be done by parallelizing execution, for instance on graphics processing units (GPUs), and eventually using a more powerful platform than Gro, such as CellModeller developed by Rudge et al. (52) or our own TB under construction (53). The next question is the ability to grow and polarize secondary cores. It should be relatively easy to make a new wheel sprout at the extremity of a stick, simply by letting the limb's LEADER cell at the tip (the red cell pushed away in all simulations) become the new central leader of a homeostatic core, while the nearby FLESH cells become POP1 and POP2 and proliferate around it. Then, precursor positioning and limb growth would proceed as usual, leading to Class-III organisms (Fig. 1).

Developmental complexity increases sharply with Class IV, however, as these organisms require long-range correlated positional information across multiple cores to be able to display limbs that are globally parallel or aligned. This would require an additional component, MorphoBrick 4, dedicated to communication among crown cells in order to allow them to detect their bearing $(\mathrm{N}$, $\mathrm{S}, \mathrm{W}$ or $\mathrm{E}$ ) with respect to the root limb that gave rise to the core, and control the start of the wave of differentiation so that the N/S polarization axis is not random. Finally, the gap between Class IV and Class V seems relatively less challenging as it would only require a new ability for two limbs growing toward each other to meet at their tips and merge into one. 
Polymorphism, Polyphenism and Evolution. The basis of the morphogenetic engineering effort consists of a developmental process executed by a "morphogenetic engine" (here a bacterial simulator, or a Petri dish), whose purpose is to take in input an agent-level genotype $G$ and transform it into a collective phenotype $P$ in output. It does this via a swarm of $G$-carrying agents that coordinate their behavior and differentiate (by direct peer-to-peer signaling and/or indirect stigmergic cues deposited in the environment), move and self-assemble (by division and/or aggregation), and collectively construct an architecture. We believe that our rational-design methodology based on compositional building blocks such as MorphoBricks is particularly appropriate when targeting a generative family of shapes or classes of shapes.

To achieve polymorphism inside a given species (here a "class"), a crafted $G \mapsto P$ mapping like ours must offer the possibility of internal parametrization of $G$ (regulatory parameters such as thresholds, rates, and frequencies) so that it can give rise to different "traits" in $P$ (here different limbs). This is similar to the classical laws of population genetics, schematically corresponding to the concepts of alleles or SNPs in the DNA. When grown freely, in absence of environmental perturbation, the effect of genotypic parametrization is to offer a family of different "breeds" within the same species, as in Mendel's peas or Darwin's pigeons (here the various star shapes of Class I, and letter shapes of Class II).

Moreover, under a given (possibly parametrized) $G \mapsto P$ mapping, the development process itself should also be sensitive to, and modifiable by environmental conditions. External conditions or stimuli encountered by one individual during its growth, whether mechanical or signaling (here obstacles and chemicals), should be able to influence the outcome, giving rise to polyphenism. This is the level of $P$, for which natural analogies can be found more readily in plants than animals. Trees can be spruced, pruned, bent, arranged, sculpted, etc., whether intentionally by humans (bonsais, espaliers, topiaries) or spontaneously when faced with adverse or favorable conditions (wind, rocks, light, humidity). The same "gardening" metaphor could apply here to morphogenetic synthetic biology.

Ultimately, however, the rigidity of pure top-down genome design, parametrized or not, is 
incompatible with the highly adaptive living material that is supposed to implement it. This is why beyond the quantitative variations underlying polymorphism and polyphenism, we also need to rely on the most obvious and powerful force of qualitative variations and true innovation in biology: evolution - which consists of random mutations and nonrandom selection. In simulation, it means the use of evolutionary computation methods, such as genetic programming, accompanied by a diversity-preserving fitness function to encourage novelty search. In a wet-lab setup, it may also mean letting the bacteria re-evolve by themselves in vivo. This should be the topic of a forthcoming article.

\section{— METHODS}

Model of Bacterial Behavior Ontology. Because prokaryotic cells generally possess a simpler organization and genome than eukaryotic cells, they are the favorite object of synthetic biology experiments and applications. Our first step was to define an abstract model of virtual prokaryote based on E. coli. This bacterium contains embedded sensors and effectors that allow it to assess and modify its internal state and environmental conditions (Fig. 12). Through the sensors, it can detect whether concentrations of specific internal proteins or external ligands cross certain thresholds, including signals emitted by its internal clock. For simplicity, we chose an explicit clocking scheme, assuming that its realization in terms of biochemical oscillators is well known. Through the effectors, it is able to release or absorb external signals, and accumulate or deplete internal proteins. Naturally, our bacterium must also be able to divide, rest, and disappear. Division is not instantaneous but the outcome of a "proliferating state" in which the cell enters: in order to divide, it needs to elongate and double its size by executing an internal growth cycle. Death, however, is an atomic action directly triggered by the cell itself. Besides division and death, a bacterium can also move forward and/or tumble but these abilities are not be exploited in the present work. Finally, there is no direct molecular signaling between two neighboring bacteria, only indirect communication via morphogen diffusion (quorum sensing) and the mechanical constraints of 2D packing. In any case, the overall architecture emerges in an endogenous fashion. 
Bacterial dynamics, consisting of differentiation and behavior, is encapsulated in a finite state machine (FSM) whose nodes represent discrete cell types, and edges represent conditional transitions between types (Fig. 12A). Each type, denoted in the example of Fig. 12 by $D, F, Q, \ldots$ corresponds to a behavioral repertoire of possible actions $\left\{a_{1}, a_{2}, \ldots, a_{n}\right\}$. Transitions between types, i.e. differentiation events, are triggered by Boolean expressions composed of conditions $\left\{c_{1}, c_{2}, \ldots, c_{m}\right\}$ pertaining to protein concentrations and time. In reality, the FSM formalism with its explicit type nodes and differentiation edges is a convenient abstraction of a complex underlying GRN: types are a shortcut for "attractors", cliques of genes promoting and repressing each other in a cycle or a fixed point; while differentiation events represent "trajectories" in phase space between basins of attraction, followed when receiving certain signals. The present model, however, is a "grammar" of integer types and logical transitions that does not include the finer scale of genetic dynamics.

Genomic Representation. This model is represented by a declarative language of our design, Synthetic Biology Genetic Programming (SBGP), which supports the description of a "bacterial behavior ontology" (BBO) based on internal and external conditions (Fig. 12B). In an SBGP script, blocks of signals and reactions describe the chemical environment of the simulation, while the other blocks describe the bacteria's behavior. The type and transition blocks specify the nodes and edges of the FSM graph. Respectively, the behavior block describes the type-specific output actions, while the cond_transition block codes for the input Boolean expressions that trigger the transitions (see full specifications in Supporting Information, "SBGP language").

The latter two blocks are defined on the basis of two groups of commands: effector primitives calling the cell's actions, and sensor primitives allowing the designer to interface with the state of internal and external sensors (both tuned by parameters). More precisely:

- Type-specific actions: when a cell belongs to a given type, it executes behaviors associated with this type until it differentiates again or dies. These belong to three categories: (1) external interactions, by which cells can absorb or diffuse morphogens in the environment 
(typically, EmitSignal); (2) internal interactions, by which cells can accumulate or deplete internal proteins; and (3) mechanical rules, making cells tumble, proliferate, rest, etc.- - and die (typically, Divide, StopDivide).

- Type-differentiation conditions: a transition to a new type is triggered when certain conditions are fulfilled. These also belong to three categories: (1) chemical conditions, monitoring whether the concentration level of an external signal or internal protein is above or below a threshold parameter (typically, GreaterThreshold and LessThreshold); (2) stochastic conditions, introducing probabilities to model rare events or stochastic noise (based on the rate keyword); and (3) "first-or-second-daughter" conditions, allowing asymmetric divisions by letting only one of the daughter cells (chosen at random) differentiate after mitosis.

For the first implementation our model, as presented in this paper, we chose the Gro platform by Klavins et al. (5), a convenient tool offering a simple procedural language and 2D graphical simulator to model the development and behavior of microcolonies of microorganisms. In parallel, we are also currently preparing our own "synthetic biology engine" and visualization software, called Traveling Bacteria (TB) (53), which we plan on using later in replacement of Gro. Other, less physically realistic, agent-based modeling and simulation platforms, such as BacSim (54) and INDISIM (55), have also been created in the past, some of them used for example in the predictive microbiology of wastewater and food safety (56).

For now, the high-level SBGP specifications are translated into Gro commands. Note that, in this framework, division is not implemented as an abstract temporal event, whether regular or probabilistic, but is triggered by the physical growth of the rod-shape bacterium when it reaches a certain length. Spatial elongation is modeled in Gro by a differential equation on the volume (not detailed here) (5). The end effect is that division happens on average every 200 time steps, i.e. 20 min with a time step $\Delta t$ representing $0.1 \mathrm{~min}$. In our simulations, all cells grow-and-divide in the same way, except when they execute the custom command StopDivide, which sets their growth rate to zero. 


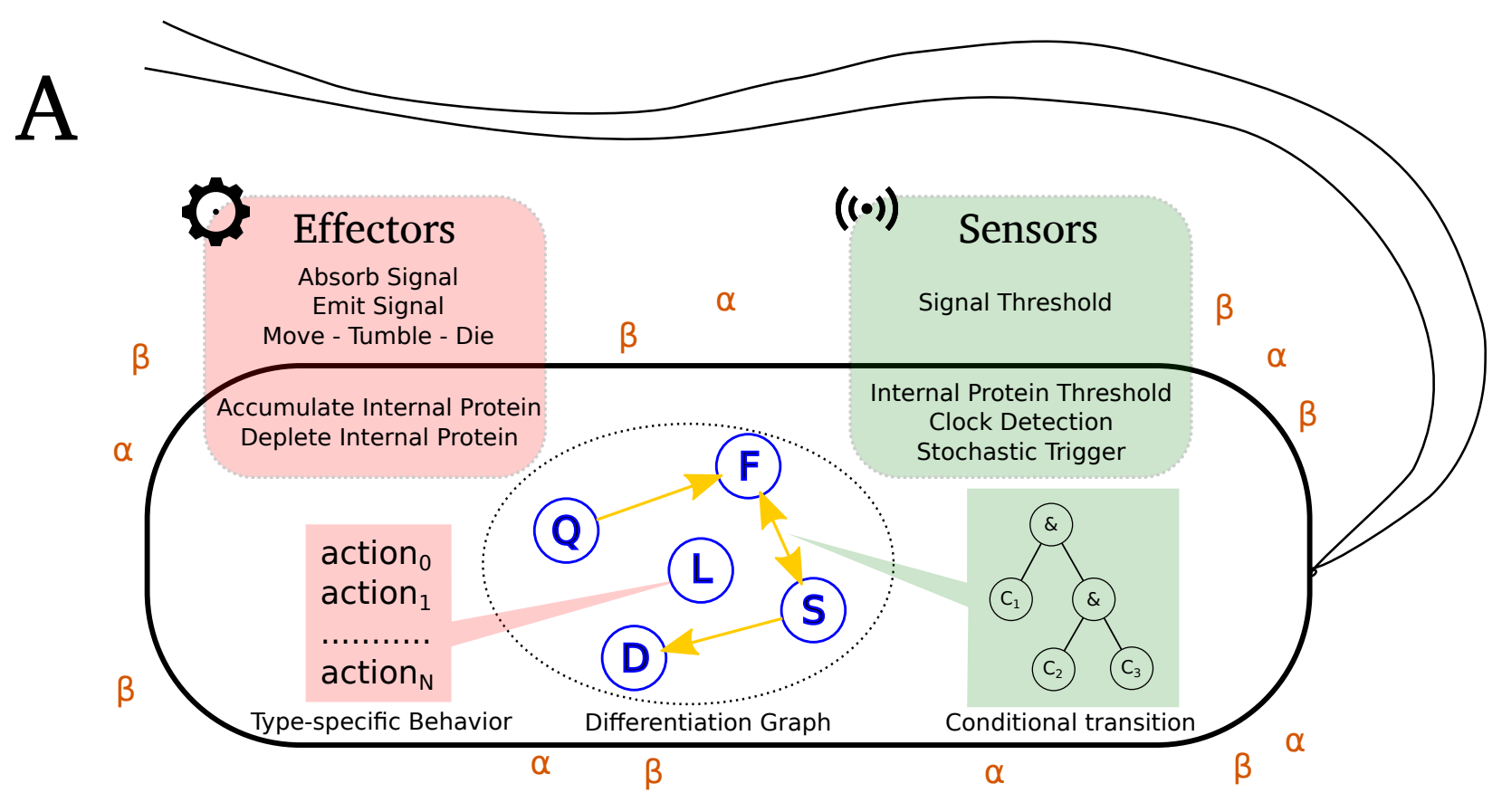

B

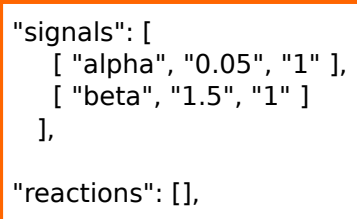

\begin{tabular}{|c|c|}
\hline $\begin{array}{l}\text { "signals": [ } \\
\text { [ "alpha", "0.05", "1" ], } \\
\text { [ "beta", "1.5", "1" ] } \\
\text { ], } \\
\text { "reactions": [], }\end{array}$ & 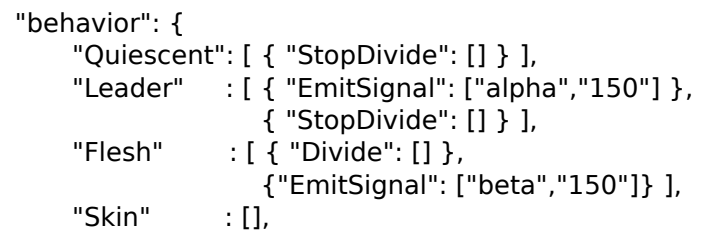 \\
\hline $\begin{array}{l}\text { "type": [ "Quiescent", "Leader", } \\
\text { "Flesh", "Skin", "Dead" ] }\end{array}$ & $:[\{$ "Die",[] $\}]$ \\
\hline $\begin{array}{l}\text { "transition": [ } \\
\text { [ "NA_", "NA_", "CQF", "NA_", "NA_" ], } \\
\text { [ "NA_", "NA_", "NA_", "NA_", "NA-" ], } \\
\text { [ "NA_",, "NA_", "NA_", "CFS", "NA_" ], } \\
\text { [ "NA_", "NA_", "CSF", "NA_", "CSD" ], } \\
\text { [ "NA_", "NA_", "NA_", "NA_", "NA_" ] } \\
\text { ] }\end{array}$ & $\begin{array}{l}\text { "cond_transition": }\{ \\
\text { "CQF" : }\{\text { "GreaterThreshold" : ["alpha","35"] \}, } \\
\text { "CFS" : \{ "LessThreshold" : ["alpha","0.5"] \}, } \\
\text { "CSF" : }\{\text { "GreaterThreshold" : ["alpha","0.5"] \}, } \\
\text { "CSD" : }\{\text { "LessThreshold" : ["alpha","0.8"] \} } \\
\text { \} }\end{array}$ \\
\hline
\end{tabular}

Figure 12. Virtual E. coli genome and associated symbolic representation. (A) Individual cell features are encapsulated in an abstract genome consisting of a type-differentiation graph (finite-state machine). It is composed of logical conditions controlling the transitions on the edges, and actions specific to each node. Conditions are based on the state of the cell's sensors, here detecting whether certain chemical concentrations have crossed given thresholds. Actions represent a "bacterial behavior ontology" (BBO) comprising mechanical and chemical events such as dividing or emitting a signal. Thus when a condition is fulfilled, a bacterium differentiates into a new type and starts executing its associated actions until it differentiates again or dies. (B) The Synthetic Biology Genetic Programming (SBGP) declarative language represents the genome and includes several related blocks. In this script example: signals and reactions describe the chemical environment of the simulation ( $\alpha$ and $\beta$ molecules), type and behavior define the genomic graph's nodes, transition and cond_transition define its edges. 
Higher-Level Biological Functions. The SBGP scripting language was also designed to handle more complex cellular mechanisms by providing useful shortcut keywords compiled into lengthier sets of Gro instructions. In addition to the low-level sensor and effector primitives, four higherlevel functions are currently available to the modeler: self-election of leaders, refractory periods, internal oscillatory clocks, and centerlines between gradients. An essential feature supporting the production of complex morphologies is the ability for seed cells to pop up spontaneously without the need to predefine them. This property is implemented here by a rare stochastic event generator inside each bacterium, handled via the Gro instruction rate. Differentiation events are analogous to chemical reactions at low rate constants, which are very slow on average and give rise to discrete occurrences with high variability over time and populations. Using this mechanism, the probability to observe two bacteria differentiate (nearly) simultaneously is very small.

The second feature is based on Gro's edge detection example (5), in which the detection or emission of a signal is followed by a refractory period where the signal cannot be sensed or released again. This results in wave propagation typical of excitable media, which is another form of communication among bacteria in an assembly. A traveling wave can carry a message fast across neighboring cells, which can react and handle it as needed. Overall, the main advantage of a refraction mechanism is better temporal and spatial accuracy of interbacterial communication than mere concentration gradients, especially when used in conjunction with an internal oscillatory mechanism (see third feature below), as is the case in the MorphoBrick-3 stage of our model. Refraction in each bacterium relies on a counter marking the elapsed time since the last detection or emission event occured. Once the counter reaches a specific threshold, the disabled function is enabled again. Biologically, this internal counter variable represents a simple model of temporary saturation of membrane receptors or blocking of molecular transport and exocytosis. For example, the refractory period may represent the time it takes for receptors or vesicles to be replenished. In SBGP, a typical refraction command is LessThresholdRefracted.

Another important high-level mechanism handled by an SBGP command is the ability to monitor the state of an internal oscillator in each cell. Here we also made the strong assumption that 
cellular oscillators are already globally synchronized at the population level, instead of modeling each of them independently, coupling them and waiting for phase-locking to emerge. This "clockon-the-wall" approach is justified by the fact that global synchrony and quorum sensing are rather common phenomena. Naturally, the advantage of this shortcut assumption is to greatly decrease the dimensionality of parameter space, the behavioral complexity of the model and the computing time of the simulation, in order to reach morphogenetic outputs more quickly.

Finally, to reach Class II and higher classes of programmable multibacterial shapes in SBGP, cells need to be able to self-identify as belonging to an equatorial boundary between two emitting sources of gradients. This mechanism is a well-known morphogenetic "design pattern" used in amorphous/spatial computing $(7,57)$ and morphogenetic engineering $(26)$. It is handled here by a Boolean function MidPlane $(A, B, \theta)$ where $A, B$ are the two emitting sources of $\alpha$ and $\beta$, and $\theta$ is a threshold tuning the width of the centerline, which translates to a double inequality $0 \leq$ $|[\alpha]-[\beta]| \leq \theta$ in the script language.

Hierarchical Composition. Ultimately, the compositional aspect of the MorphoBrick framework could be implemented in hierarchical gene circuits, as outlined by Doursat (41). Taking after the early regulatory cascade of Drosophila, which goes from global protein gradients to the precise positioning of appendages, the idea is to rely on "identity genes" expressed in specific regions of the multicellular colony (similar to "imaginal discs"). Identity genes switch on dedicated subcircuits that further subdivide regions into subregions and shape their expansion by differential proliferation. New positional information also arises inside regions via the propagation of local gradients, and so on. In sum, the morphogenesis of a complex articulated structure can ultimately proceed by recursive, multiscale refinement of details-except that the absolute scale remains the same (the individual bacterium), it is only the relative scale that decreases while the multicellular organism grows, as pointed out by Coen (46).

Chemical Environment. The bacterial population is immersed in a diffusive chemical environment represented by a discrete diffusion grid in Gro, which relies on Euler's method to solve the 
associated partial differential equations. The Gro environment is non-toroidal and its size typically set to $160 \mu \mathrm{m} \times 160 \mu \mathrm{m}$ in our experiments. The underlying diffusion grid consists of square locations of size $(0.5 \mu \mathrm{m})^{2}$ each, i.e. $320 \times 320$ of them. Cell coordinates are real-valued and not bound to a discrete lattice. A typical E. coli rod in Gro simulations measures $2 \mu \mathrm{m} \times 1 \mu \mathrm{m}$ (real E. coli bacteria range between 0.5 and $3 \mu \mathrm{m}$ ), thus covers 8 diffusion grid squares on average. A surface area of $(160 \mu \mathrm{m})^{2}$ can contain about 12,800 cells. There are no specific boundary conditions: signals diffusing outside the grid are lost. Although a "no-flux" boundary condition would have been more realistic, the accumulation of signal along the borders is negligible and will not disturb the morphogenetic process as long as the organism remains significantly smaller than the virtual square petri dish. Chemical species $\alpha, \beta, \gamma, \ldots$ are declared in the environment by specifying both their diffusion and degradation rates (signals block in Fig. 12B). Molecules can be emitted, detected and absorbed by the bacteria. They can also react with each other via chemical equations classically expressed by reactants, products and reaction rates (athough no reaction was specified here).

\section{- SUPPORTING INFORMATION}

- SBGP LANGUAGE SPECIFICATION

- General Format

- Block Examples

- COMPILATION NOTES

- Gro Program Header

- Persistent Memory Setup

- Main Procedure

- MORPHOLOGICAL ANALYSIS

- VIDEOS

- SBGP AND GRO SOURCE CODES

- Video 1a: MorphoBrick 1 - Homeostatic Core with 2 Layers (Fig. 3)

- Video 1b: MorphoBrick 1 - Homeostatic Core with 3 Layers (Fig. 6)

- Video 2a: MorphoBrick 2 - Limb Growth Showing 1 Bend (Fig. 7)

- Video 2b: MorphoBrick 2 - Limb Growth Showing 2 Bends (Fig. 7C)

- Video 3a: MorphoBrick 3 - Precursor Cell Positioning (Fig. 11)

- Video 3b: MorphoBrick 3 - Precursor Cell Differentiation (Fig. 13)

- Video 3c: MorphoBrick 3 - Propagation \& Segmentation Mechanism (Fig. 10)

- Video 4a: Class-I Starfish Shape (Fig. 12)

- Video 4b: Class-II L-Shape (Fig. 14A) 
- Video 4c: Class-II T-Shape (Fig. 14B)

- SBGP AND GRO SOURCE CODES

- Code 1a: MorphoBrick 1 - Homeostatic Core with 2 Layers

- Code 1b: MorphoBrick 1 - Homeostatic Core with 3 Layers

- Code 2a: MorphoBrick 2 - Limb Growth

- Code 3a: MorphoBrick 3 - Precursor Cell Positioning

- Code 3b: MorphoBrick 3 - Precursor Cell Differentiation

- Code 4a: Class-I Starfish Shape

- Code 4b: Class-II L-Shape

- Code 4c: Class-II T-Shape

\section{— AUTHOR INFORMATION}

\section{Corresponding Authors}

*Emails: jonathan.pascalie@irit.fr, r.doursat@mmu.ac.uk

Notes

The authors declare no competing financial interest.

\section{- ACKNOWLEDGMENTS}

J.P. was supported by a postdoctoral fellowship from Région Paris Ile-de-France: Complex Systems Institute (ISC-PIF), Appel à projets (AAP) 2013. T.K. was supported by the SynBioTIC project grant funded by the French Agence Nationale de la Recherche (ANR-BLAN-SIMI10-LS100618-12-01), which also funded computer equipment.

\section{References}

1. Knight, T. Idempotent vector design for standard assembly of biobricks; 2003.

2. Stepney, S., Diaconescu, A., Doursat, R., Giavitto, J.-L., Kowaliw, T., Leyser, O., MacLennan, B., Michel, O., Miller, J. F., Nikolic, I., Spicher, A., Teuscher, C., Tufte, G., Vico, F. J., and Yamamoto, L. Gardening cyber-physical systems. UCNC '12: Proceedings of the 11th International Conference on Unconventional Computation and Natural Computation. 2012; pp 237-238.

3. Armstrong, R., and Spiller, N. (2010) Synthetic biology: Living quarters. Nature 467, 916-918.

4. Amos, M., Stepney, S., Doursat, R., Vico, F. J., and Rasmussen, S. (2012) TRUCE: A coordination action for unconventional computation. Int. J. Uncon. Comp. 8, 333-337.

5. Jang, S. S., Oishi, K. T., Egbert, R. G., and Klavins, E. (2012) Specification and simulation of synthetic multicelled behaviors. ACS Synth. Biol. 1, 365-374.

6. Giavitto, J.-L., and Michel, O. (2002) The topological structures of membrane computing. Fund. Informaticae 49, 123-145.

7. Beal, J., and Bachrach, J. (2006) Infrastructure for engineered emergence on sensor/actuator networks. IEEE Intell. Syst. 21, 10-19. 
8. Cameron, D. E., Bashor, C. J., and Collins, J. J. (2014) A brief history of synthetic biology. Nat. Rev. Microbiol. 12, 381-390.

9. Monod, J., and Jacob, F. General conclusions: teleonomic mechanisms in cellular metabolism, growth, and differentiation. Cold Spring Harbor Symposia on Quantitative Biology. 1961; pp 389-401.

10. Westerhoff, H. V., and Palsson, B. O. (2004) The evolution of molecular biology into systems biology. Nat. Biotechnol. 22, 1249-1252.

11. Bray, D. (1995) Protein molecules as computational elements in living cells. Nature 376, 307-312.

12. McAdams, H. H., and Shapiro, L. (1995) Circuit simulation of genetic networks. Science 269, 650-656.

13. Gardner, T. S., Cantor, C. R., and Collins, J. J. (2000) Construction of a genetic toggle switch in Escherichia coli. Nature 403, 339-342.

14. Elowitz, M. B., and Leibler, S. (2000) A synthetic oscillatory network of transcriptional regulators. Nature 403, 335-338.

15. Weiss, R., and Basu, S. The device physics of cellular logic gates. NSC-1: The First Workshop of Non-Silicon Computing. Boston, Massachusetts. 2002.

16. Rackham, O., and Chin, J. W. (2005) A network of orthogonal ribosome- mRNA pairs. Nat. Chem. Biol. $1,159-166$.

17. Ham, T. S., Lee, S. K., Keasling, J. D., and Arkin, A. P. (2006) A tightly regulated inducible expression system utilizing the fim inversion recombination switch. Biotechnol. Bioeng. 94, 1-4.

18. Palsson, B. (2000) The challenges of in silico biology. Nat. Biotechnol. 18, 1147-1150.

19. Weiss, R., and Knight Jr, T. F. DNA Computing; Springer-Verlag, 2001; pp 1-16.

20. You, L., Cox, R. S., Weiss, R., and Arnold, F. H. (2004) Programmed population control by cell-cell communication and regulated killing. Nature 428, 868-871.

21. Basu, S., Gerchman, Y., Collins, C. H., Arnold, F. H., and Weiss, R. (2005) A synthetic multicellular system for programmed pattern formation. Nature 434, 1130-1134.

22. Tabor, J. J., Salis, H. M., Simpson, Z. B., Chevalier, A. A., Levskaya, A., Marcotte, E. M., Voigt, C. A., and Ellington, A. D. (2009) A synthetic genetic edge detection program. Cell 137, 1272-1281.

23. Liu, C., Fu, X., Liu, L., Ren, X., Chau, C. K., Li, S., Xiang, L., Zeng, H., Chen, G., Tang, L.-H., Lenz, P., Cui, X., Huang, W., Hwa, T., and Huang, J.-D. (2011) Sequential establishment of stripe patterns in an expanding cell population. Science 334, 238-241.

24. Amos, M. (2014) Population-based microbial computing: a third wave of synthetic biology? Int. J. Gen. Syst. 43, 770-782.

25. Turing, A. M. (1952) The chemical basis of morphogenesis. Philos. Trans. R. Soc., B 237, 37-72.

26. Doursat, R., Sayama, H., and Michel, O. (2013) A review of morphogenetic engineering. Nat. Comput. $1-19$. 
27. Doursat, R., and Sánchez, C. (2014) Growing fine-grained multicellular robots. Soft Robot. 1, 110-121.

28. Bongard, J. C., and Pfeifer, R. Morpho-functional Machines: The New Species; Springer-Verlag, 2003; pp 237-258.

29. Stanley, K. O., and Miikkulainen, R. (2003) A taxonomy for artificial embryogeny. Artif. Life 9, 93-130.

30. Kowaliw, T., Grogono, P., and Kharma, N. Bluenome: A Novel Developmental Model of Artificial Morphogenesis. Genetic and Evolutionary Computation (GECCO). 2004; pp 93-104.

31. Doursat, R. Facilitating evolutionary innovation by developmental modularity and variability. Proceedings of the 11th annual conference on genetic and evolutionary computation (GECCO). 2009; pp 683690.

32. Doursat, R., Sánchez, C., Dordea, R., Fourquet, D., and Kowaliw, T. In Morphogenetic Engineering: Toward Programmable Complex Systems; Doursat, R., Sayama, H., and Michel, O., Eds.; Understanding Complex Systems; Springer-Verlag, 2012; pp 275-311.

33. Joachimczak, M., Kowaliw, T., Doursat, R., and Wróbel, B. (2013) Evolutionary design of soft-bodied animats with decentralized control. Artif. Life Robot. 18, 152-160.

34. Rubenstein, M., Cornejo, A., and Nagpal, R. (2014) Programmable self-assembly in a thousand-robot swarm. Science 345, 795-799.

35. Komosinski, M. Virtual worlds; Springer-Verlag, 2000; pp 214-224.

36. Lipson, H., and Pollack, J. B. (2000) Automatic design and manufacture of robotic lifeforms. Nature 406, 974-978.

37. Cussat-Blanc, S., Pascalie, J., Mazac, S., Luga, H., and Duthen, Y. Morphogenetic Engineering; Springer-Verlag, 2012; pp 353-381.

38. Cussat-Blanc, S., and Pollack, J. (2014) Cracking the egg: Virtual embryogenesis of real robots. Artif. Life 20, 361-383.

39. Eggenberger, P. Evolving morphologies of simulated 3D organisms based on differential gene expression. ECAL '97: Proceedings of the Fourth European Conference on Artificial Life. 1997; pp 205-213.

40. Disset, J., Cussat-Blanc, S., and Duthen, Y. Self-organization of Symbiotic Multicellular Structures. ALIFE XIV: Proceedings of the 14th International Conference on the Simulation and Synthesis of Living Systems. 2014; pp 3-5.

41. Doursat, R. (2006) The growing canavas of biological development: multiscale pattern generation on an expanding lattice of gene regulatory networks. InterJournal 1809.

42. Reuillon, R., Leclaire, M., and Rey-Coyrehourcq, S. (2013) OpenMOLE, a workflow engine specifically tailored for the distributed exploration of simulation models. Future Gener. Comp. Sy. 29, 1981-1990.

43. Rudge, T. J., Federici, F., Steiner, P. J., Kan, A., and Haseloff, J. (2013) Cell polarity-driven instability generates self-organized, fractal patterning of cell layers. ACS Synth. Biol. 2, 705-714.

44. Schlosser, G., and Wagner, G. P. Modularity in development and evolution; University of Chicago Press, 2004. 
45. Carroll, S. B., Grenier, J. K., and Weatherbee, S. D. From DNA to diversity: molecular genetics and the evolution of animal design; John Wiley \& Sons, 2013.

46. Coen, E. The Art of Genes: How Organisms Make Themselves: How Organisms Make Themselves; Oxford University Press, 1999.

47. Waters, C. M., and Bassler, B. L. (2005) Quorum sensing: cell-to-cell communication in bacteria. Annu. Rev. Cell Dev. Biol. 21, 319-346.

48. Dagkessamanskaia, A., Moscoso, M., Hénard, V., Guiral, S., Overweg, K., Reuter, M., Martin, B., Wells, J., and Claverys, J.-P. (2004) Interconnection of competence, stress and CiaR regulons in Streptococcus pneumoniae: competence triggers stationary phase autolysis of ciaR mutant cells. Mol. Microbiol. 51, 1071-1086.

49. Pascalie, J., Lobjois, V., Luga, H., Ducommun, B., and Duthen, Y. Checkpoint Orientated Cell Cycle Modeling Issues in Simulation of Synchronized Situation. ALIFE XIII: Proceedings of the 13th International Conference on the Simulation and Synthesis of Living Systems. 2012; pp 465-472.

50. Danino, T., Mondragón-Palomino, O., Tsimring, L., and Hasty, J. (2010) A synchronized quorum of genetic clocks. Nature 463, 326-330.

51. Peter, I. S., and Davidson, E. H. (2009) Modularity and design principles in the sea urchin embryo gene regulatory network. FEBS Lett. 583, 3948-3958.

52. Rudge, T. J., Steiner, P. J., Phillips, A., and Haseloff, J. (2012) Computational modeling of synthetic microbial biofilms. ACS Synth. Biol. 1, 345-352.

53. Potier, M. Integration of levels of organization in a language dedicated to the modeling of complex systems. Chapter 3: Traveling Bacteria. Ph.D. thesis, Université Paris-Est Créteil (UPEC), France, 2016.

54. Kreft, J.-U., Booth, G., and Wimpenny, J. W. (1998) BacSim, a simulator for individual-based modelling of bacterial colony growth. Microbiology+ 144, 3275-3287.

55. Ginovart, M., Lopez, D., and Valls, J. (2002) INDISIM, an individual-based discrete simulation model to study bacterial cultures. J. Theor. Biol. 214, 305-319.

56. Esser, D. S., Leveau, J. H., and Meyer, K. M. (2015) Modeling microbial growth and dynamics. Appl. Microbiol. Biotechnol. 99, 8831-8846.

57. Nagpal, R. Complex Engineered Systems; Springer-Verlag, 2006; pp 291-306. 
Graphical TOC Entry

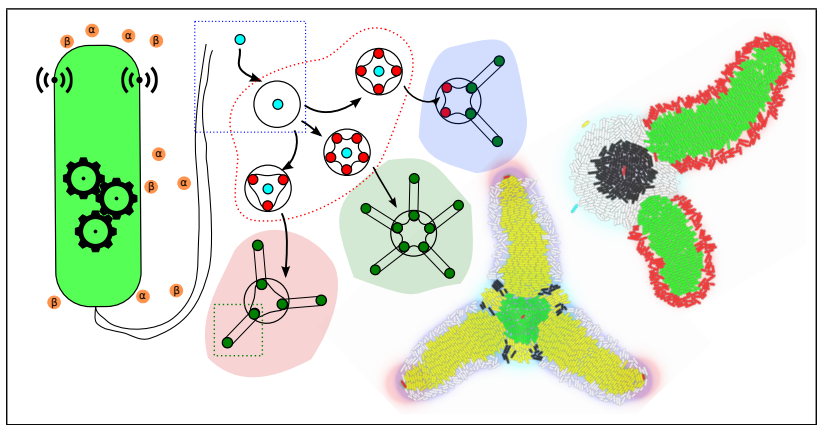

